This is the peer-reviewed author's accepted manuscript of:

Haile-Selassie Y, Melillo SM, Vazzana A, Benazzi S, Ryan TM. A 3.8-million-year-old hominin cranium from Woranso-Mille, Ethiopia. Nature (2019 Aug 28)

The final published version is available online at:

https://doi.org/10.1038/s41586-019-1513-8

https://www.nature.com/articles/s41586-019-1513-8

This version is subjected to Nature terms for reuse that can be found at:

http://www.nature.com/authors/policies/license.html\#terms 


\title{
A 3.8-million-year-old hominin cranium from Woranso-Mille, Ethiopia
}

Yohannes Haile-Selassie ${ }^{1,5}$, Stephanie M. Melillo ${ }^{2,5}$, Antonino Vazzana ${ }^{3}$, Stefano Benazzi ${ }^{3}$ \& Timothy M. Ryan ${ }^{4}$

\begin{abstract}
The cranial morphology of the earliest known hominins in the genus Australopithecus remains unclear. The oldest species in this genus (Australopithecus anamensis, specimens of which have been dated to 4.2-3.9 million years ago) is known primarily from jaws and teeth, whereas younger species (dated to 3.5-2.0 million years ago) are typically represented by multiple skulls. Here we describe a nearly complete hominin cranium from Woranso-Mille (Ethiopia) that we date to 3.8 million years ago. We assign this cranium to $A$. anamensis on the basis of the taxonomically and phylogenetically informative morphology of the canine, maxilla and temporal bone. This specimen thus provides the first glimpse of the entire craniofacial morphology of the earliest known members of the genus Australopithecus. We further demonstrate that A. anamensis and Australopithecus afarensis differ more than previously recognized and that these two species overlapped for at least 100,000 years - contradicting the widely accepted hypothesis of anagenesis.
\end{abstract}

The absence of cranial remains of Australopithecus species that are older than 3.5 million years has limited our understanding of the evo-lutionary history of this genus. Here we describe a nearly complete hominin cranium, dated to approximately 3.8 million years (Myr) ago, that fills a crucial gap in the hominin fossil record. The specimen shows a morphology that is more primitive than that of any previ-ously known Australopithecus cranium, including features that link early Australopithecus to the Mio-Pliocene genera Sahelanthropus and Ardipithecus. Derived features are concentrated in the face, appearing in an unexpected combination that is variably shared with A. afarensis and paralleling the morphology that is present in later australopiths.

Discoveries of hominin fossils in the past three decades have resulted in the naming of numerous new taxa, including the earliest species known thus far1-11. These discoveries have added to our understand-ing of human evolution by pushing the hominin fossil record into the Miocene epoch $2-4,10$, and by showing possible taxonomic diversity5,9, wider geographical distributions1, the presence of multiple forms of bipedalism6,12 and a major adaptive shift associated with the origin of the genus Australopithecus12. At the same time, these discoveries raise important questions related to hominin taxonomy and systematics $9,13,14$. Although most questions emanate from the fragmentary (mostly dentognathic) nature of the fossil record and small sample size, some issues relate to the absence of fossils from critical time periods and skeletal elements informative in systematics.

The Woranso-Mille study area, located in the Afar region of Ethiopia, has become one of the most important sites and has yielded homi-nin fossils from a poorly known period of the mid-Pliocene epoch. Since 2005, fieldwork at Woranso -Mille has been aimed at answer-ing questions about mid-Pliocene hominin diversity and testing the hypothesized ancestor-descendant relationship of A. anamensis and A. afarensis. In this regard, Woranso-Mille hominin fossils have shown that more than one hominin species was present during the mid-Pliocene epoch (at least A. afarensis and an as-yet-unnamed species that is represented by the Burtele foot)6,15. Another species, Australopithecus deyiremeda, has also been named on the basis of fossils from 3.5-3.3-Myr-old deposits, even though this has been contested13. The 3.8-3.6-Myr-old hominin fossils-which are mostly dentognathic - from Woranso -Mille have also corroborated the proposed ancestor-descendant relationship between A. anamensis (4.2-3.9 Myr ago) and A. afarensis (3.7-3.0 Myr ago)16,17. Fieldwork in 2016 resulted in the recovery of a nearly complete cranium of an early hominin (Fig. 1). This cranium (MRD-VP-1/1, hereafter MRD) was dated to $3.8 \mathrm{Myr}$ ago 18 and hence comes from a critical period close to $4 \mathrm{Myr}$ ago, during which the craniofacial morphology of early hominins is almost completely unknown. To our knowledge, MRD is the first specimen to shed light on the full cranial anatomy of the earliest known australopiths. Details of the discovery and preservation of the specimen are provided in Supplementary Note 1. Missing parts and minor distortions of this specimen were reconstructed digitally (Extended Data Figs. 1-5, Methods and Supplementary Notes 2-5).

\section{Morphological descriptions}

The MRD cranium has a well-developed sagittal crest and a robustly built, long and prognathic face. Its preserved right canine is among the largest known for early hominins, especially in its mesiodistal dimension. These features suggest that MRD represents a male, despite the fact that the specimen is very small in overall size. This individual had reached an advanced developmental age. The preserved postcanine teeth are heavily worn (almost to the cervico-enamel junction), except for the $\mathrm{M}^{3}$. Most of the sutures are obliterated on the ectocranial surface and the alveolar bone shows age-related remodelling.

The specimen is readily identifiable as a hominin by the following morphological features: the canine is reduced in size compared to nonhuman apes and shows a strong lingual basal tubercle; the mastoids are inflated; the nuchal plane is more horizontal than in non-human apes; and the inion, which is coincident with the opisthocranion, lies near the level of the Frankfort horizontal plane. At the same time, the small cranial capacity, highly prognathic face, extensive pneumatization and other features discussed below indicate that MRD represents a hominin that is more primitive than A. afarensis.

Analyses of the maxilla and dentition

The MRD canine is relatively large (compared to other homin-ins) and shows mesiodistal elongation. Basic dimensions align the 

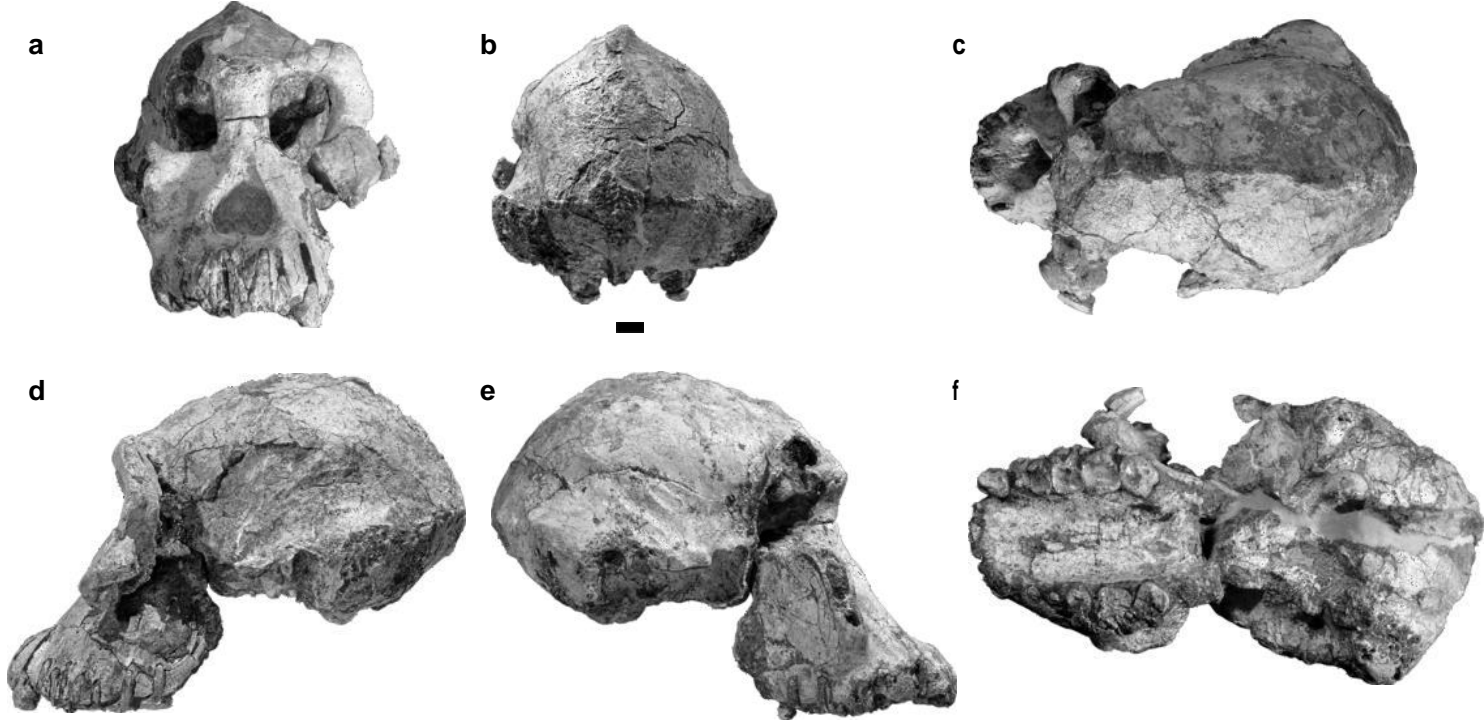

Fig. 1 | The MRD-VP-1/1 cranium. a, Anterior view. b, Posterior view. c, Superior view. d, Left lateral view. e, Right lateral view. f, Inferior view. The specimen is oriented in Frankfort horizontal plane. Scale bar, $1 \mathrm{~cm}$.

specimen with A. anamensis or more-primitive hominins ${ }^{19-22}$ (Fig. 2a, b and Supplementary Note 6). Furthermore, a number of qualitative features are shared between MRD and A. anamensis upper canines (Fig. 2c). The mesial and distal basal tubercles (crown 'shoulders') are short, equidistant from the cervico-enamel junction and occlusally divergent. The degree of mesiodistal elongation and short shoulders are particularly important, because they are among the few distinctive features described for A. anamensis that are not shared by A. afarensis or Ardipithecus ramidus ${ }^{19,23}$. Short mesial shoulders are, how-ever, present in upper canines attributed to Ardipithecus kadabba ${ }^{24}$ and Orrorin tugenensis ${ }^{10}$. Additional primitive features shared among the canines of MRD, A. anamensis and other earlier hominins are present on the lingual crown face. The lingual ridge is large, blunt and mesially offset, which relegates the mesial lingual fossa to a small slit-like structure at the mesial-most extent of the lingual face ${ }^{25}$. A wear facet is present on the lingual face in MRD, KNM-KP 58309 and some A. ramidus specimens. This feature may have been produced

through non-honing contact with an asymmetric and mesiobuccally bulging $\mathrm{P}_{3}$.

Little can be said about the morphology of the other teeth, owing to damage and wear. However, even though the incisor crowns are bro-ken off, the remaining parts of the crowns and the shape of the roots indicate that the incisors were vertically implanted. A wide (approxi-mately 4$\mathrm{mm}) \mathrm{I}^{2}-\mathrm{C}$ diastema is present in MRD. This feature is absent in the Sahelanthropus tchadensis cranium ${ }^{3}$, but is variably present in $A$. afarensis and has also been documented in A. ramidus (for example, ARA-VP-6/500-115) ${ }^{26}$. The MRD canine and midface are posteriorly inclined, which is a notable difference from some of the A. anamensis specimens $7,19,21$ (Extended Data Fig. 6 and Supplementary Note 6). The $\mathrm{M}^{3}$ crown is sub-triangular in outline and rotated in the dental row such that the protocone-hypocone crown wall faces in the distolingual direction. The latter feature occurs commonly in A. anamensis specimens, but less so in A. afarensis.

The MRD maxillary dental arcade is relatively narrow and slightly Ushaped (Supplementary Note 6). The canine is aligned with the postcanine tooth row (Extended Data Fig. 7). The inferolateral margin of the nasal aperture in MRD is smooth and rounded owing to the con-fluence of a pronounced canine jugum with the nasal margin. This is also the condition that is found in A. anamensis specimens ${ }^{19,21,25}$. Most A. afarensis maxillae have thin lateral margins and sharp infero-lateral corners $^{27}$. The MRD palate is very deep, a morphology that is conventionally considered to be derived. The depth at the $\mathrm{M}^{1} / \mathrm{M}^{2}$ level (17.4 mm) exceeds all known A. anamensis and A. afarensis specimens
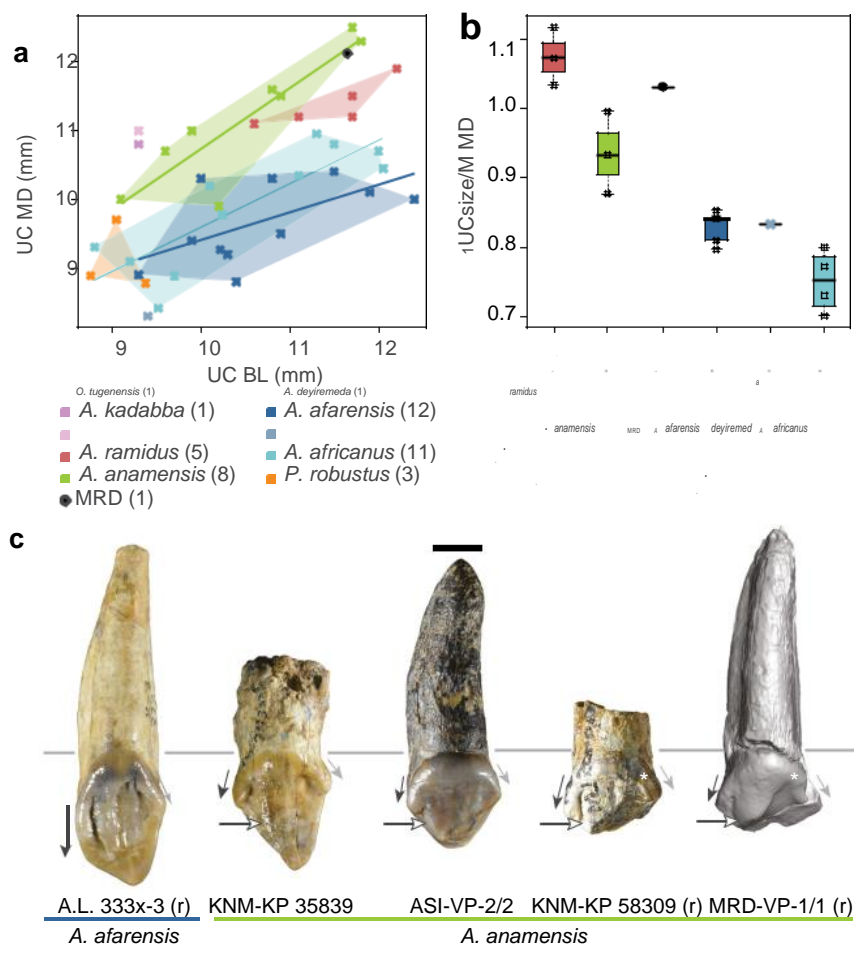

Fig. 2 Comparisons of upper canine morphology. a, Upper canine basal dimensions. Least- squares regression lines are shown for A. anamensis, $A$. afarensis and A. africanus. BL, buccolingual; MD, mesiodistal; UC, upper canine. b, Relative canine size $\left(\sqrt{ }(\mathrm{MD} \times \mathrm{BL}) / \mathrm{M}^{1} \mathrm{MD}\right)$. Box plots or individual observations are shown. The box plots show the centre line (median), box limits (upper and lower quartiles), whiskers (range) and individual values (squares). Taxa are arranged chronologically, showing the temporal trend of decreasing relative canine size. Sample sizes $(n)$ are reported in parentheses after the species names. c, Lingual face of the canine crown in A. afarensis and A. anamensis at sequential wear stages. The length and orientation of the mesial and distal basal tubercles are illustrated by black and grey arrows, respectively. White arrows indicate

a slit-like mesial lingual fossa and white asterisks mark the extension of a wear facet onto the lingual crown face. Canines are aligned at the cervical enamel line (dashed grey line). All specimens are shown as right teeth and (r) indicates images that have been reversed. 
a

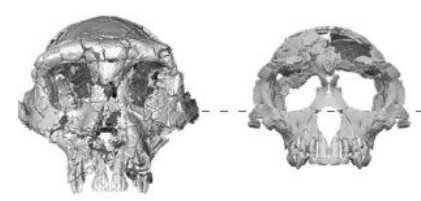

b

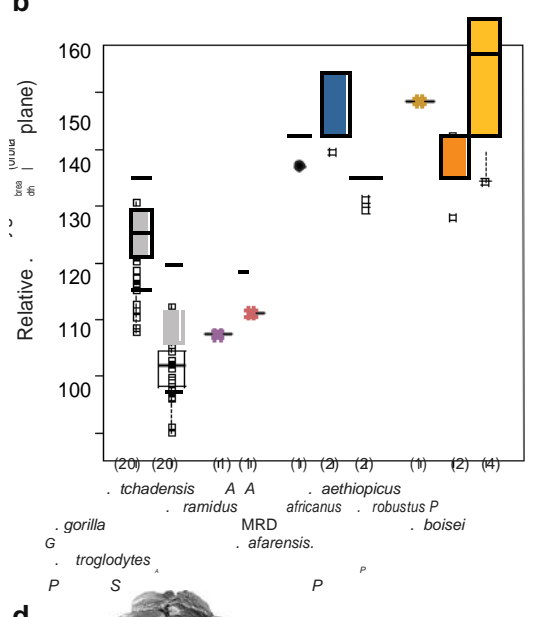

d

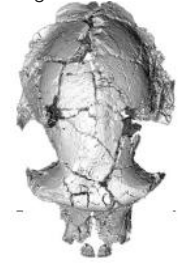

e

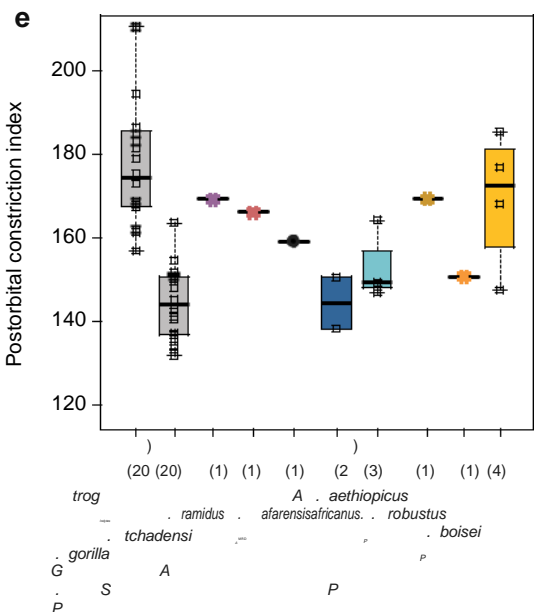

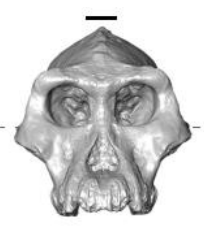
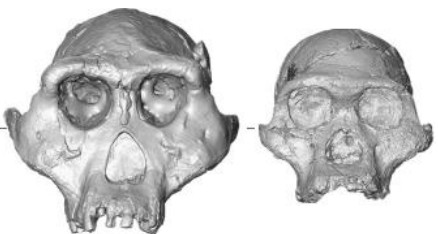

C

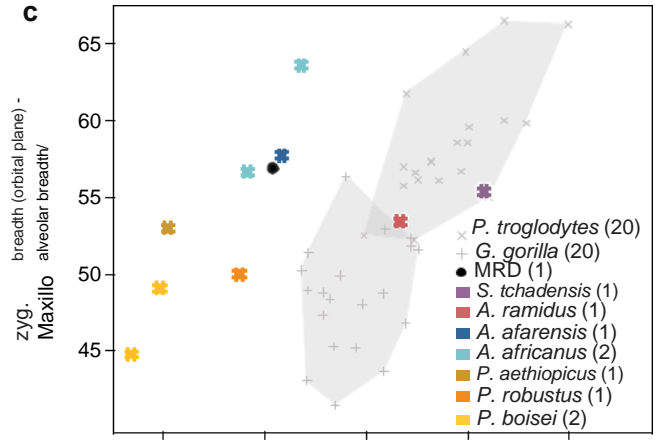

708090100110 Sup. facial breadth/bizyg. breadth (orbital plane)
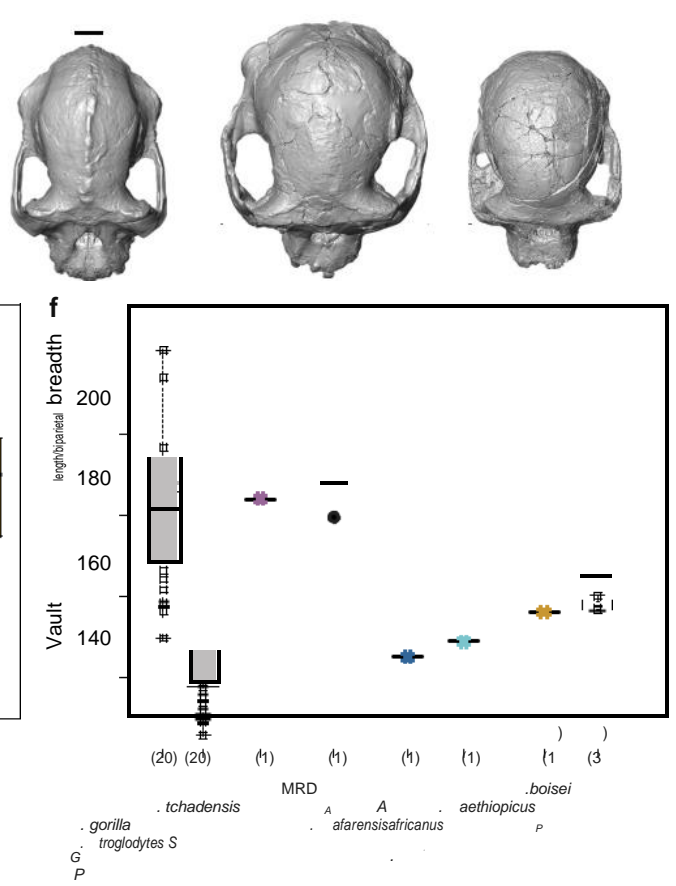

Fig. 3 Structure of the face and neurocranium. a, Anterior view of early hominin crania. Left to right: $S$. tchadensis (TM 266-01-60-1), composite A. ramidus reconstruction, MRD, A. afarensis (A.L. 444-2), A. africanus (Sts 5). Specimens are oriented in Frankfort horizontal and aligned at orbitale (dashed grey line). b, Bizygomatic (bizyg.) breadth (in the orbital plane) relative to biorbital breadth. G. gorilla, Gorilla gorilla; P. aethiopicus, Paranthropus aethiopicus; P. boisei, Paranthropus boisei; P.troglodytes, Pan troglodytes. c, Bivariate plot of indices that quantify the 'facial mask' shape. d, Superior view of early hominin crania (in the same order as in a). Specimens are oriented in Frankfort horizontal and aligned at glabella (dashed grey line). e, Index of postorbital constriction (superior facial breadth/postorbital constriction). f, Index that quantifies the braincase shape. Box plot definitions are as in Fig. 2 and sample sizes are indicated in the figure panels. Scale bars,

$2 \mathrm{~cm}$. Images of A. ramidus and $S$. tchadensis in a, d were generated, with permission, from CT scans that have previously been published 26,45 and falls among the deepest palates of A. africanus and Paranthropus robustus (Supplementary Note 7).

\section{Analysis of the face}

Australopiths share a pattern of facial structure that is characterized by a broad zygomatic region combined with a relatively narrow upper face. This produces an upward-tapering outline (the 'hexagonal facial mask' $)^{27-29}$. Although the hexagonal mask is shared among non-robust and robust australopiths, $S$. tchadensis, A. ramidus and non-human apes exhibit relatively broader upper faces and lack pronounced zygo-matic expansion $^{26,27}$. MRD is similar to other australopiths and distinct from earlier hominins in possessing a broad midface and narrow upper face (Fig. 3a-c). There are facial similarities that are specifically shared between MRD and $A$. afarensis. The external contour of the orbits in MRD and $A$. afarensis is squared off superolaterally and the lateral border of the orbit widens inferiorly. Facial hafting is similar between MRD and non-robust australopiths: the frontal bone is slightly inclined, which positions the face below the braincase, and no post-toral sulcus or frontal trigon is present (Extended Data Fig. 8).
The MRD face is particularly long supero-inferiorly. This morphology stands in stark contrast to the short and gracile face reconstructed for A. ramidus. However, comparisons of facial robusticity must consider sexual dimorphism: MRD, A.L. 444-2 and TM 266-01-60-1 probably represent male individuals, whereas the face of the A. rami$d u s$ composite represents a female ${ }^{26}$. Thus some degree of difference in facial robusticity is expected. The currently available fossil sample does not permit the disentanglement of the morphological differences that are due to sexual dimorphism from those that are taxonomically diagnostic. Furthermore, it has previously been suggested ${ }^{26}$ that the short face of the composite reconstruction of A. ramidus is not representative of the species. The MRD face is also strongly prognathic, both in the mid-face and subnasally (Extended Data Fig. 8). The projection of the mid-face in MRD is comparable to $S$. tchadensis, but MRD lacks upper facial projection ${ }^{3,26}$

There are some aspects of the MRD face that are reminiscent of $A$. africanus and robust australopiths, and that have traditionally been considered to be derived. The MRD zygomaticoalveolar crest is nearly straight and rises steeply from the alveolar margin. As a result, the 
Table 1 | Craniofacial metrics of MRD-VP-1/1 and other early hominins

\begin{tabular}{|c|c|c|c|c|c|c|c|}
\hline & & S. tchadensis & A. ramidus & A. afarensis & A. afric & & \\
\hline & MRD-VP-1/1 & $\begin{array}{l}\text { TM 266-01-060-1 } \\
\text { reconstruction }\end{array}$ & $\begin{array}{l}\text { ARA-VP-6/500 } \\
\text { reconstruction }\end{array}$ & A.L. 444-2 & Sts 5 & Sts 71 & Stw 505 \\
\hline \multicolumn{8}{|l|}{ Cranial dimensions } \\
\hline Vault length (g-op) & 149.2 & 173 & - & 167 & 146 & 129 & - \\
\hline Maximum cranial length (pr-op) & 205.0 & 211 & $162.5 \pm 3$ & 215 & 181 & 164 & - \\
\hline ba-b height & 94.9 & 86 & 79.5 & & 101 & - & - \\
\hline Biparietal breadth $^{a}$ & 83.1 & $90^{p}$ & $91-92.5^{j}$ & 115 & 98 & - & - \\
\hline Maximum cranial breadth ${ }^{b}$ & 116.3 & 128 & 110.5 & 139 & 108 & - & 123 \\
\hline Biporion breadth & 108.9 & $114.2^{p}$ & 98.5 & 132 & 107 & 105 & $(126)$ \\
\hline Postorbital breadth ${ }^{c}$ & $61.9^{k}$ & 65 & 62 & 77 & 64 & 56 & 71 \\
\hline Occipital upper scale arc (I-op) & 26.1 & - & - & 29 & 39 & 33 & - \\
\hline Occipital upper scale angle (I-op) & 70.1 & $(63)^{p}$ & - & 70 & 80 & - & - \\
\hline Occipital lower scale arc (op-o) & 56.4 & - & - & 41 & 31 & 38 & - \\
\hline Occipital lower scale angle (op-o) & 43.1 & 36 & - & 25 & 40 & - & - \\
\hline Nuchal plane length (i-0) & 54.1 & $42.8^{p}$ & - & - & 31 & 38 & - \\
\hline \multicolumn{8}{|l|}{ Facial dimensions } \\
\hline Superior facial breadth (fmt-fmt) & 98.5 & 110 & 103 & $(116)$ & 94 & $(92)$ & - \\
\hline Bizygomatic breadth at the orbital plane ${ }^{d}$ & 122.0 & 108.3 & $107.5^{\mathrm{j}}$ & 142 & 120 & 110 & - \\
\hline Bimaxillary breadth (zm-zm) & $92 \pm 2^{1}$ & - & 83.5 & - & 106 & 96 & - \\
\hline Maxillo-alveolar breadth & 69.5 & 60 & 59 & 82 & 68 & 70 & - \\
\hline Superior facial height (n-pr) & $91.1^{k}$ & 75 & $61.5 \pm 3$ & 100 & 77 & 73 & 91 \\
\hline Vertical facial height ${ }^{e}$ & 105 & $99.5^{p}$ & - & 113 & 86 & 88 & 110 \\
\hline Malar height (or-zm) & $(34)^{\prime}$ & 26 & 18 & & 26 & 27 & $31^{m}$ \\
\hline Orbitoalveolar height $^{\dagger}$ & 59.3 & $46.8^{p}$ & 32.5 & 66 & 51 & 47 & 62 \\
\hline Masseteric height $\mathrm{g}$ & 38 & $26.8^{p}$ & $18.2^{\mathrm{j}}$ & 32.3 & 32.1 & 31 & - \\
\hline ns-pr height & 30.7 & 21.5 & - & & 29 & 27 & 29 \\
\hline ns-pr angle & 48.9 & 50 & - & 39 & 37 & 47 & 50 \\
\hline Index of palate protrusion ${ }^{\mathrm{h}}$ & 69.4 & 47 & $53.2^{j}$ & 55 & 68 & 43 & - \\
\hline Sellion-pr angle & 54.7 & $(60)^{p}$ & $52.5^{\mathrm{i}}$ & 65 & 53 & 65 & $(58)$ \\
\hline Sellion-ns angle & 58.1 & - & - & 76 & 66 & 72 & 66 \\
\hline Biorbital breadthi & 82.8 & $91^{n}$ & $90.5^{n}$ & 95 & 85 & 79 & $97^{\circ}$ \\
\hline Interorbital breadth (mf-mf) & $23.1^{k}$ & 23 & $16.6^{\mathrm{i}}$ & 19 & 20 & 20 & 18.6 \\
\hline Orbit breadth (mf-ek) & 31.5 & 37.5 & 37 & $40^{n}$ & 34 & 34 & 39 \\
\hline Orbit height & 31.3 & 35 & 32 & 39 & 29 & 31 & 37 \\
\hline Nasal aperture breadth ${ }^{i}$ & $24.1^{k}$ & 26 & 18.5 & 25 & 26 & 25 & 29 \\
\hline Nasal aperture height (rhi-ns) & $38.7^{k}$ & 32 & - & 37 & 24 & 24 & 28 \\
\hline Internal palate length (ol-sta) & $73.6^{k}$ & - & - & 75 & 65.3 & - & - \\
\hline
\end{tabular}

Measurements of MRD are taken from the symmetrized reconstruction, or from the original specimen where the concerned region is undistorted (as indicated). Distances are reported in mm. Angles are measured between the concerned chords and Frankfort horizontal planes and reported in degrees. Values in parentheses or with ' \pm ' indicate estimated measurements; '-' indicates unavailable data. Comparative data were assembled from previous studies ${ }^{27-29,42-45}$ or collected by the authors. Definitions for the osteometric point abbreviations can be found in a previously published study ${ }^{46}$. aDefinition follows 'maximum parietal breadth' of ref. 43

bEither bimastoid or bisupramastoid, depending on specimen morphology. For MRD, it is bisupramastoid.

${ }^{\mathrm{M}}$ Measurement $9(1)$ of ref. ${ }^{46}$ and measurement 21 of ref. ${ }^{27}$ were used.

dMeasurement 52 of ref. ${ }^{27}$ was used.

eMeasurement 53 of ref. ${ }^{27}$ was used.

Measurement 58 of ref. ${ }^{27}$ was used.

${ }^{8}$ Measurement 59 of ref. ${ }^{27}$ was used.

hSee definition in refs 27,46 .

Maximum breadth parallel to Frankfort horizontal plane.

iMeasurements provided by G. Suwa (personal communication).

${ }^{k}$ Measurement of the original specimen.

'The zygomaxillary suture is obliterated. The position of $\mathrm{zm}$ was estimated at the medial third of the zygomatic tubercle.

${ }^{m}$ Minimum malar height as described in ref. ${ }^{43}$. This measurement is equivalent to or- $\mathrm{zm}$ in some specimens and $1-3 \mathrm{~mm}$ less than or-zm in others.

nfmo-fmo, typically 1-3 mm less than maximum breadth.

ek-ek.

PMeasurement provided by F. Guy (personal communication).

anterior origin of the masseter muscle (the zygomatic tubercle) is superiorly positioned and the tubercle faces inferolaterally. The opposite pattern, in which the crest is low and arched, is traditionally considered to be primitive, because it is shared among A. ramidus, A. afarensis and African apes30,31. A ratio of the masseteric height relative to the vertical facial height shows that MRD is similar to A. africanus and robust australopiths in exhibiting a high ratio that indicates a relatively superior masseter position. By contrast, S. tchadensis, A. ramidus and A. afarensis share lower values (Supplementary Note 8). The masseter ori-gin is also anteriorly positioned in MRD, similar to robust australopiths

(Supplementary Note 8). However, the anterior position is achieved in a unique way. The infra-orbital malar region is concave in MRD, rather than flat as in A. afarensis. This concavity shifts the lateral portion of the zygomatic body (including the tubercle) more anteriorly than the zygomatic root (Fig. 3d). As a result, the central portion of the face is concealed by the zygomatic bones in lateral view, despite the zygomatic root being positioned at M1 (Extended Data Fig. 8).

\section{Description of the neurocranium}

The MRD neurocranium retains a number of primitive features and sutural patterns. It retains the asterionic notch and the squamosal suture is low and only slightly arched. It is anteroposteriorly elongated and narrow across the parietals - an unusual shape that is shared exclu-sively with S. tchadensis (Fig. 3d, f). Our preliminary estimate of the cranial capacity is 365-370 c.c., similar to S. tchadensis. This value is smaller than the smallest female individuals of A. afarensis27,32. The pronounced postorbital constriction (as indicated by large index value) seen in MRD is shared to various degrees by other hominins, including the mostprimitive species, S. tchadensis and A. ramidus. However, A. afarensis differs as it has a relatively wider postorbital region (Fig. 3d, e). In MRD, the sagittal crest begins more anteriorly than in the crania of most hominins and continues posteriorly to merge with the nuchal lines.

The temporal squama of MRD is heavily pneumatized and also has a small external acoustic meatus. The A. anamensis temporal fragment 
(KNM-KP 29281B) shares these features. Although the dimensions of the MRD external acoustic meatus cannot be measured precisely owing to damage, the better-preserved left side is approximately $8.0 \mathrm{~mm}$ vertically and $8.9 \mathrm{~mm}$ anteroposteriorly with an estimated ellipse area of $55.9 \mathrm{~mm} 2$, which is larger than KNM-KP 29281B7 (40 mm2). However, the value of MRD falls in the lower range of A. afarensis27 (47.7-109.3 $\mathrm{mm} 2$; mean $=79.7 \mathrm{~mm} 2 ; \mathrm{n}=4$ ). A posterior view reveals a bell-shaped outline, a compound temporal-nuchal crest and a 'bare area' morphology that is comparable to A. afarensis27 (Extended Data Fig. 9). The MRD mastoids are inflated, although they are positioned slightly above the cranial base. As a result, the contour of the cranial base is convex, similar to the condition in Pan and to some degree to the condition in A. africanus, but in contrast to A. afarensis (Extended Data Fig. 9). Another primitive feature of the cranial base is the great length of the nuchal plane. In MRD, the nuchal plane length-standardized by bimastoid breadth - is longer than that of TM266-01-60-1 (Table 1).

\section{Taxonomy and phylogenetic relationships}

The morphological comparisons presented above indicate that MRD is best attributed to A. anamensis. Although most of the similarities between MRD and A. anamensis are plesiomorphic, MRD presents many features that distinguish A. anamensis from A. afarensis: a rel-atively large and mesiodistally elongated upper canine, a small exter-nal acoustic meatus, a rounded lateral nasal margin with contribution from the canine jugum, a relatively narrow palate and an upper canine aligned with the postcanine row. Morphology of the MRD upper canine is particularly important in taxonomic attribution, because it displays features that appear to be apomorphic in A. anamensis (basally posi-tioned crown shoulders and pronounced mesiodistal elongation of the crown). The most notable differences between MRD and the previ-ously known A. anamensis hypodigm include the greater inclination of the MRD canine and the greater depth of the palate.

However, our comparisons of canine inclination indicate that the magnitude of dif-ference between MRD and other A. anamensis specimens is consistent with intraspecific variability (Extended Data Fig. 6 and Supplementary Note 6).

Additional plesiomorphic features of A. anamensis are revealed for the first time in MRD. The nuchal plane is very long and postorbital constriction is pronounced. The shape of the neurocranium in superior view has notable similarities to that of S. tchadensis. However, aspects of the mid- and upper face show clear affinity to A. afarensis. The facial mask is hexagonal and the superolateral corner of the orbits is 'squared off' in a distinctive manner. Facial hafting in MRD is similar to the condition shared between A. afarensis and A. africanus (that is, inclined frontal lacking a post-toral sulcus).

A. anamensis is consistently recognized as being phylogenetically positioned between A. ramidus and A. afarensis7,19,20,25,33,34. Although this idea has both chronological and morphological support, the ana-tomical composition of the previously known hypodigm is limited to comparisons of jaws and teeth. Our understanding is thus primarily based on where A. anamensis fits with regard to documented trends in canine reduction and the development of masticatory robusticity. The discovery of MRD presents an opportunity to consider the phylogenetic position of A. anamensis using craniofacial morphology.

We conducted phylogenetic analyses by augmenting two charac-ter matrices published by independent observers27,33 with data from MRD (Supplementary Notes 9 and Supplementary Table 1). In one iteration of these analyses, MRD was treated as one specimen within the larger A. anamensis hypodigm. The resulting cladograms depict A. anamensis as the sister taxon to A. afarensis and later hominins, thus reinforcing the widely accepted topology (Extended Data Fig. 10a, b). Results are similar when MRD was treated as a separate operational taxonomic unit (Extended Data Fig. 10c-g, Supplementary Note 9 and Supplementary Table 1). Together, these analyses indicate that the phylogenetic position of A. anamensis is consistent, even when differ-ent anatomical regions are considered (that is, dentognathic and/or craniofacial morphologies) and despite inter-observer differences in character list composition and scoring.

Although the addition of craniofacial observations does not change the accepted topology, it does alter the implied pattern of morphologi-cal evolution. A. anamensis is generally portrayed as a primitive exten-sion of A. afarensis; this idea has been supported by the apparent lack of derived features in the previously known hypodigm19,35. However, the craniofacial morphology of MRD suggests that A. anamensis possessed a number of derived features, including the presence of topographic relief in the infraorbital region, a superiorly and anteriorly positioned masseter origin, and potentially additional features (Extended Data Fig. 10h, i and Supplementary Note 9). Notably, the presence of pro-nounced postorbital constriction in MRD and more primitive homi-nins confirms the previous suggestion that A. afarensis is derived in showing reduced postorbital constriction27,35. It also further confirms that the 3.9-Myr-old frontal fragment from the Middle Awash, Ethiopia (BEL-VP-1/1)36,37, is derived with regard to postorbital constriction and thus probably belongs to A. afarensis27,38 (Supplementary Note 10). Together, the secure dating of BEL-VP-1/1 and MRD indicate that A. afarensis and A. anamensis overlapped in the Afar Triangle for at least 100,000 years.

\section{Discussion}

The 3.8-Myr-old cranium from Woranso-Mille elaborates some of the outstanding questions in Pliocene hominin evolution and fills a major gap in the fossil record. The morphologies of the MRD canine, maxilla and temporal region suggest that this specimen represents A. anamensis.

The hypothesis that A. anamensis and A. afarensis constitute a single evolving chronospecies was based on limited apomorphic features in A. anamensis, but is mostly due to perceived temporal trends in morphology in four time-successive samples of the two species from Kanapoi, Allia Bay, Laetoli and Hadar35. However, the lack of fossil hominins from 3.93.7 Myr ago in general and the lack of complete crania of A. anamensis in particular have been major impediments to fully test this hypothesis. The addition of MRD to the A. anamensis hypodigm changes our understanding of the relationship between the two taxa.

With MRD assigned to A. anamensis, it indicates that A. anamensis can be clearly distinguished from A. afarensis such that the latter species may not have been a result of 'phyletic transformation within an unbranched lineage'35. Furthermore, the fact that MRD shares some neurocranial and facial morphological features with younger taxa such as A. africanus and Paranthropus - albeit considered here to be more likely to have been caused by parallel evolution - is worth further investigation in the future, as it may have considerable bearing on the origin of A. africanus and its relationship with A. afarensis. More work is also needed to better understand the geology and Pliocene palaeo-geography of the Afar region $^{18}$ and establish a refined taxonomy of the Woranso-Mille hominins from 3.8 to 3.4 Myr ago.

On the basis of the currently available fossil evidence, it appears that there were at least four time-successive but allopatric A. anamensis populations (Woranso-Mille, Allia Bay, Asa Issie and Kanapoi) that showed variable cranial and dentognathic morphology. Although the cranial morphology of A. anamensis was poorly known thus far, the 3.8Myr-old MRD from Woranso-Mille is morphologically similar to the species from Kanapoi and Asa Issie (4.2-4.1 Myr ago). However, it is unlikely that an A. anamensis population represented by MRD gave rise to A. afarensis, as MRD postdates BEL-VP-1/1, which now appears to be the earliest known representative of $A$. afarensis with an age of approximately 3.9 Myr. Although their taxonomic affinity is still controversial $^{39}$, it is possible that the few approximately 4.0-Myr-old teeth from Fejej, Ethiopia, might also belong to A. afarensis ${ }^{40,41}$. These observations can be tested with the recovery of crania from Kanapoi, Asa Issie and Allia Bay and further comparisons to MRD. In sum-mary, although MRD and other discoveries from Woranso-Mille do not falsify the proposed ancestor-descendant relationship between A. anamensis and A. afarensis, they indicate that $A$. afarensis may not have evolved from a single ancestral population. Most importantly, MRD shows that despite the widely accepted hypothesis of anagenesis, A. afarensis did not appear as a result of phyletic transformation. It also shows that at least two related hominin species co-existed in eastern Africa around 3.8 Myr ago, further lending support to mid-Pliocene hominin diversity.

1. Brunet, M. et al. Australopithecus bahrelghazali, une nouvelle espèce d'Hominidé ancien de la région de Koro Toro (Tchad). C. R. Acad. Sci. IIA 322, 907-913 (1996) 
2. Brunet, M. et al. New material of the earliest hominid from the Upper Miocene of Chad. Nature 434, 752-755 (2005).

3. Brunet, M. et al. A new hominid from the Upper Miocene of Chad, Central Africa. Nature 418, 145-151 (2002).

4. Haile-Selassie, Y. Late Miocene hominids from the Middle Awash, Ethiopia. Nature 412, 178-181 (2001).

5. Haile-Selassie, Y. et al. New species from Ethiopia further expands Middle Pliocene hominin diversity. Nature 521, 483-488 (2015).

6. Haile-Selassie, Y. et al. A new hominin foot from Ethiopia shows multiple Pliocene bipedal adaptations. Nature 483, 565-569 (2012).

7. Leakey, M. G., Feibel, C. S., McDougall, I. \& Walker, A. New four-millionyear-old hominid species from Kanapoi and Allia Bay, Kenya. Nature 376, 565-571 (1995)

8. Leakey, M. G., Feibel, C. S., McDougall, I., Ward, C. \& Walker, A. New specimens and confirmation of an early age for Australopithecus anamensis. Nature 393, 62-66 (1998).

9. Leakey, M. G. et al. New hominin genus from eastern Africa shows diverse middle Pliocene lineages. Nature 410, 433-440 (2001).

10. Senut, B. et al. First hominid from the Miocene (Lukeino formation, Kenya). C. R. Acad. Sci. IIA 332, 137-144 (2001).

11. White, T. D., Suwa, G. \& Asfaw, B. Australopithecus ramidus, a new species of early hominid from Aramis, Ethiopia. Nature 371, 306-312 (1994).

12. White, T. D. et al. Ardipithecus ramidus and the paleobiology of early hominids. Science 326, 64-86 (2009).

13. Wood, B. \& K Boyle, E. Hominin taxic diversity: fact or fantasy? Am. J. Phys. Anthropol. 159, 37-78 (2016).

14. Haile-Selassie, Y., Melillo, S. M. \& Su, D. F. The Pliocene hominin diversity conundrum: do more fossils mean less clarity? Proc. Natl Acad. Sci. USA 113, 6364-6371 (2016).

15. Haile-Selassie, Y. et al. Dentognathic remains of Australopithecus afarensis from Nefuraytu (Woranso-Mille, Ethiopia): comparative description, geology, and paleoecological context. J. Hum. Evol. 100, 35-53 (2016).

16. Haile-Selassie, Y. Phylogeny of early Australopithecus: new fossil evidence from the Woranso-Mille (central Afar, Ethiopia). Phil. Trans. R. Soc. Lond. B 365, 3323-3331 (2010).

17. Haile-Selassie, Y., Saylor, B. Z., Deino, A., Alene, M. \& Latimer, B. M. New hominid fossils from Woranso-Mille (Central Afar, Ethiopia) and taxonomy of early Australopithecus. Am. J. Phys. Anthropol. 141, 406-417 (2010).

18. Saylor, B. Z. et al. Age and context of new mid-Pliocene hominin cranium from Woranso-Mille, Ethiopia. Nature https://doi.org/10.1038/s41586-0191514-7 (2019)

19. Ward, C. V., Leakey, M. G. \& Walker, A. Morphology of Australopithecus anamensis from Kanapoi and Allia Bay, Kenya. J. Hum. Evol. 41, 255-368 (2001).

20. Ward, C. V., Manthi, F. K. \& Plavcan, J. M. New fossils of Australopithecus anamensis from Kanapoi, West Turkana, Kenya (2003-2008). J. Hum. Evol. 65, 501-524 (2013).

21. Ward, C. V., Plavcan, J. M. \& Manthi, F. K. New fossils of Australopithecus anamensis from Kanapoi, West Turkana, Kenya (2012-2015). J. Hum. Evol. https://doi.org/10.1016/j.jhevol.2017.07.008 (2017)

22. Manthi, F. K., Plavcan, J. M. \& Ward, C. V. New hominin fossils from Kanapoi, Kenya, and the mosaic evolution of canine teeth in early hominins. S. Afr. J. Sci. 108, 724 (2012).

23. Suwa, G. et al. Paleobiological implications of the Ardipithecus ramidus dentition. Science 326, 69-99 (2009).

24. Haile-Selassie, Y., Suwa, G. \& White, T. D. Late Miocene teeth from Middle Awash, Ethiopia, and early hominid dental evolution. Science 303, 1503 1505 (2004).

25. White, T. D. et al. Asa Issie, Aramis and the origin of Australopithecus. Nature 440, 883-889 (2006).

26. Suwa, G. et al. The Ardipithecus ramidus skull and its implications for hominid origins. Science 326, 68-68e7 (2009).

27. Kimbel, W. H., Rak, Y. \& Johanson, D. C. The Skull of Australopithecus afarensis (Oxford Univ. Press, 2004).

28. Guy, F. et al. Morphological affinities of the Sahelanthropus tchadensis (Late Miocene hominid from Chad) cranium. Proc. Natl Acad. Sci. USA 102, 18836-18841 (2005).

29. Rak, Y. The Australopithecine Face (Academic, 1983).

30. Kimbel, W. H. \& Rak, Y. Australopithecus sediba and the emergence of Homo: Questionable evidence from the cranium of the juvenile holotype $\mathrm{MH}$ 1. J. Hum. Evol. 107, 94-106 (2017).

31. Kimbel, W. H., White, T. D. \& Johanson, D. C. Cranial morphology of Australopithecus afarensis: a comparative study based on a composite reconstruction of the adult skull. Am. J. Phys. Anthropol. 64, 337-388 (1984).

32. Kimbel, W. H. \& Rak, Y. The cranial base of Australopithecus afarensis: new insights from the female skull. Phil. Trans. R. Soc. Lond. B 365, 3365-3376 (2010).

33. Strait, D. S. \& Grine, F. E. Inferring hominoid and early hominid phylogeny using craniodental characters: the role of fossil taxa. J. Hum. Evol. 47, 399452 (2004).

34. Dembo, M., Matzke, N. J., Mooers, A. Ø. \& Collard, M. Bayesian analysis of a morphological supermatrix sheds light on controversial fossil hominin relationships. Proc. R. Soc. Lond. B 282, 20150943 (2015).

35. Kimbel, W. H. et al. Was Australopithecus anamensis ancestral to A afarensis? A case of anagenesis in the hominin fossil record. J. Hum. Evol. 51, 134-152 (2006).

36. Asfaw, B. The Belohdelie frontal: new evidence of early hominid cranial
37. Renne, P. R. et al. Chronostratigraphy of Mio-Pliocene Sagantole Formation, Middle Awash Valley, Afar rift, Ethiopia. Bull. Geol. Soc. Am. 111, 869-885 (1999).

38. Kimbel, W. H., Johanson, D. C. \& Rak, Y. The first skull and other new discoveries of Australopithecus afarensis at Hadar, Ethiopia. Nature 368, 449451 (1994).

39. Ward, C. V. Taxonomic affinity of the Pliocene hominin fossils from Fejej, Ethiopia. J. Hum. Evol. 73, 98-102 (2014).

40. Fleagle, J. G., Rasmussen, D. T., Yirga, S., Bown, T. M. \& Grine, F. E. New hominid fossils from Fejej, Southern Ethiopia. J. Hum. Evol. 21, 145-152 (1991).

41. Kappelman, J. et al. Age of Australopithecus afarensis from Fejej, Ethiopia.J. Hum. Evol. 30, 139-146 (1996).

42. Kimbel, W. H., Johanson, D. C. \& Coppens, Y. Pliocene hominid cranial remains from the Hadar Formation, Ethiopia. Am. J. Phys. Anthropol. 57 453-499 (1982). 
43. Wood, B. Koobi Fora Research Project: Hominid Cranial Remains Vol. 4 (Clarendon, 1991)

44. Lockwood, C. A. \& Tobias, P. V. A large male hominin cranium from Sterkfontein, South Africa, and the status of Australopithecus africanus. J. Hum. Evol. 36, 637-685 (1999).

45. Zollikofer, C. P. et al. Virtual cranial reconstruction of Sahelanthropus tchadensis. Nature 434, 755-759 (2005).

46. Martin, R. \& Knussman, R. Anthropologie: Handbuch der vergleichenden Biologie des Menschen Vol. 1 (Gustav Fischer, 1988).

Acknowledgements We thank the Authority for Research and Conservation of Cultural Heritage (ARCCH) for permission to conduct field and laboratory workthe Afar people of Woranso-Mille and the Mille District administration for their hospitality; the project's fieldwork crew members for their tireless support of field activities; The National Museum of Kenya, National Museum of Tanzania, Ditsong Museum and the Evolutionary Studies Institute of South Africa for access to original hominin specimens in their care; Max Planck Institute for Evolutionary Anthropology for access to comparative hominin computed tomography scan data; T. White, G. Suwa and B. Asfaw for access to the original A. ramidus material and for providing images and unpublished measurements; M. Brunet and F. Guy for images and unpublished measurements of $S$. tchadensis; W. H. Kimbel for informative discussions and for access to a surface model of the A.L. 444-2 cranium; D. Lieberman (the Peabody Museum (Harvard)), R. Beutel (Phyletisches Museum Jena), C. Funk (Museum für Naturkunde), M. Tocheri (National Museum of Natural History (Smithsonian)), U. Olbrich-Schwarz (the Max Planck Institute for Evolutionary Anthropology) for the use of computed tomography scans of extant apes; A. Girmaye, M. Endalamaw, Y. Assefa, T. Getachew, S. Melaku and G. Tekle of ARCCH for access to the fossil collections housed in the Paleoanthropology Laboratory in Addis Ababa; T. Stecko from the Penn State Center for Quantitative Imaging for assistance with computed tomography scanning; and N. Meisel and D. N. Kaweesa (Made By Design Lab (Pennsylvania State University)) for assistance in 3D printing. This research was supported by grants from the US National Science Foundation (BCS-1124705, BCS-1124713, BCS-1124716, BCS-1125157 and BCS-1125345) and The Cleveland Museum of Natural History. Y.H.-S. was also supported by W. J. and L. Hlavin, T. and K. Leiden, and E. Lincoln. S.B. was supported by the European Research Council (ERC) under the European Union's Horizon 2020 research and innovation programme (ERC-724046-SUCCESS; http://www.erc-success.eu). S.M.M. was supported by the Max Planck Institute for Evolutionary Anthropology, Department of Human Evolution.

Author contributions Y.H.-S. and S.M.M. conducted fieldwork and collected data. T.M.R. scanned the specimen using computed tomography. T.M.R., S.B. and A.V. performed the three-dimensional reconstruction with contributions from Y.H.-S. and S.M.M. Y.H.-S. and S.M.M. performed the comparative analysis. S.M.M. and Y.H.-S. wrote the paper with contributions from T.M.R., S.B. and A.V 


\section{Methods}

Computed tomography. The MRD cranium was scanned using a GE v|tome|x L300 industrial multiscale microcomputed tomography scanner at the Pennsylvania State University Center for Quantitative Imaging. The cranium was scanned with source energy settings of $180 \mathrm{kV}$ and $420 \mu \mathrm{A}, 0.2-\mathrm{mm} \mathrm{Al}$ filter, 2,400 projections, 5 frames averaged per projection and a detector timing of $500 \mathrm{~ms}$. The data were reconstructed with an isotropic voxel size of $75 \mu \mathrm{m}$. The data were segmented and a three-dimensional isosurface of the external surface of the fossil was created in Avizo v.9.3 (ThermoFisher Scientific)

Reconstruction of the MRD cranium. Reconstruction of MRD involved recon-structing the facial skeleton and neurocranium, integrating missing parts, recon-structing the endocast and estimating cranial capacity. These procedures are described below.

Digital reconstruction of the facial skeleton. The surface obtained was divided into neurocranial (endocranium and ectocranium) and facial portions. In the original fossil, these two parts were glued together, but this intervention resulted in a mismatch between neurocranium and face, in which the facial portion is medially distorted on the left side. The misalignment appears more evident when the midsagittal plane is computed for the neurocranium (using a best-fit plane for glabella, nasion, sagittal crest and median nuchal line) and the facial portion (using a best-fit plane for rhinion, anterior nasal spine, incisive foramen and points selected on the maxillary suture). The angle computed between the two planes is $6.2^{\circ}$ (Extended Data Fig. 1a). The facial portion was rotated to align the midsagittal plane of the face with the midsagittal plane of the neurocranium ${ }^{47}$. Then, the facial portion was moved along the midsagittal plane (that is, upward/downward, forward/backward) once the alignment with the neurocranium was established (Extended Data Fig. 1b). Distorted and missing regions were restored first by mirroring the contralateral unaffected portions of the facial bones, by then using an iterative closest-point algorithm to enhance the alignment between mirrored and original homologous regions and finally by cropping and merging the reconstructed parts to the original preserved portions of MRD. Small gaps were closed using surface interpolation, maintaining - where possible - the original features of the fossil. Specifically, the left supraorbital and zygomatic bones were mirrored to reconstruct missing regions on the right side (Extended Data Fig. 1c). Then, the reconstructed right hemiface was mirrored and aligned with the left part, thus restoring the deformation of the maxilla (Extended Data Fig. 1d). Because most of the left upper teeth are preserved in the original, the mirrored right maxilla was used to correctly realign the original left maxillary dental arcade and part of the palate, thus preserving the original features of the fossil (Extended Data Fig. 1e, f). Finally, because only the third molar $\left(\mathrm{M}^{3}\right)$ is preserved on the right side, the left dental arcade (except the right $\mathrm{M}^{3}$ ) was reconstructed by mirroring the left counterpart (Extended Data Fig. 1g, h). All of the procedures described above were performed in Geomagic Design X (3D Systems Software) ${ }^{48,49}$

Symmetrization. A template consisting of 47 landmarks and 707 semilandmarks (217 curve semilandmarks and 490 surface semilandmarks) was created on the MRD cranium in Viewbox software (dHAL Software) to capture the geometry of the cranial surface (Extended Data Fig. 2a, b and Supplementary Note 2). The MRD digital model was mirrored along its midsagittal plane to create a complete, symmetric (semi)landmark configuration. The paired landmarks were reflected/ relabelled (Extended Data Fig. 2c, d) whereas the semilandmarks were allowed to slide to reduce the bending energy with respect to the original configuration. Then, both configurations were superimposed using a generalized Procrustes analysis, and the Procrustes average (consensus) shape (which is symmetrical by definition) was computed $^{47-49}$. Procrustes coordinates of the symmetric configuration were transformed to Cartesian coordinates by multiplying each (semi)landmark by the centroid size mean (the mean between the centroid size of the two configura-tions). Finally, the original MRD (semi)landmark configuration was warped into the symmetric (semi)landmark configuration using the thin plate spline (TPS) interpolation functions ${ }^{42}$ in Avizo. The surface of the MRD digital model was warped so as to minimize the bending energy of the according transformation (Extended Data Fig. 2e)

Reconstruction of the neurocranium. Further reconstruction of the neurocranium entailed creating a mirror-image copy followed by iterative closest-point superimposition of undistorted regions in order to reconstruct the right temporal process of the zygomatic arch, the left mastoid process and other incomplete parts of the basicranium (Extended Data Fig. 2f-h). We use 'MRD-sym' to refer to the surface model that results from the procedures described above (facial reconstruction, mirror imaging and symmetrization). MRD-sym was produced exclusively using data present in the original specimen.

Integration of missing parts. The rest of missing or incomplete regions of MRDsym (specifically, the orbital cavities, the zygomatic arches, the sphenoid bone, the foramen magnum and portions of the endocranium) were restored with refer-ence to morphology present in other hominin crania. Reference (semi)landmark configurations were created in Viewbox software on the crania of three different hominins (reference templates): Sts 5 (A. africanus $=650$ (semi)landmarks (Extended Data Fig. 3a, b and Supplementary Note 3)), A.L. 444-2 (A. afarensis $=438$ (semi)landmarks (Extended Data Fig. 4a, b and Supplementary Note 4)) and KNM-WT 17000 (P. aethiopicus $=312$ (semi)landmarks (Extended Data Fig. 4e, f and Supplementary Note 5)). First, the Sts 5 reference template was applied to MRD-sym to reconstruct the orbital cavities, the sphenoid bone, the foramen magnum and portions of the endocranium, as well as the zygomatic arch and small portions of the frontal process of the zygomatic bone. Semilandmarks were allowed to slide along curves and surfaces to minimize the bending energy of the TPS interpolation computed between the target and each reference, and the semilandmarks that fell in the missing regions were allowed to move without constraints (Extended Data Fig. 3c). The (semi)landmarks of the reference template were thus transformed into the corresponding (semi)landmarks of the target using the TPS functions ${ }^{50}$, whereas the surface of the reference was interpolated to minimize the bending energy of the relative transformation (Extended Data Fig. $3 \mathrm{~d}$ ). The corresponding areas of the missing regions of interest were isolated from the resulting warped surface and merged with the three-dimensional MRD-sym model (Extended Data Fig. 3e-h). We use 'MRD-Sts 5' to refer to the surface model that results from using Sts 5 as a reference specimen to integrate the missing parts. The (semi)landmark configurations created on A.L. 444-2 (Extended Data Fig. 4a, b) and KNM-WT 17000 (Extended Data Fig. 4e, f) were similarly applied to MRD-sym (Extended Data Fig. 4c, g) to create alternative reconstructions of the zygomatic arch and the frontal process of the zygomatic bone, following the same methodological procedures described above (Extended Data Fig. 4d, h). Because warping reference hominin crania might introduce slight asymmetries in the reconstructed regions, and because for $P$. aethiopicus only the left side was available to reconstruct the zygomatic arch, the left hemi-cranium of MRD-sym was mirrored along the midsagittal plane to replace the right counterpart. Care was taken to preserve the sagittal and nuchal crests of MRD-sym. The names 'MRDA.L. 444-2' and 'MRD-WT 17000' refer to the alternative reconstructions using these specimens as references.

Reconstruction of the endocast and estimation of cranial capacity. To estimate cranial capacity, the endocast was segmented manually in Avizo v.9.3. The poor greyscale contrast between the fossil and the matrix infill necessitated manual tracing of the internal endocranial bone surface. For this reconstruction, only the right half of the endocranium was used, owing to the distortion on the left side of the cranium. The hemi-endocast was then mirrored and aligned to produce a complete, undistorted and symmetrical reconstruction.

Phylogenetic analyses. Parsimony analyses were conducted using a branch and bound search algorithm. Character ordering, weighting and outgroup treatment followed the methods described in the original publications 27,33 . We report stand-ard descriptors associated with parsimony analyses, including the tree length, consistency index and retention index.

We analysed the character matrices in multiple iterations, in which MRD was treated either as its own operational taxonomic unit (OTU) or as one specimen within the larger A. anamensis hypodigm. Our scorings for MRD and any alterations to published matrices are described in Supplementary Table 1. Strait and Grine $^{33}$ included an A. anamensis OTU based on the Kenyan specimens known before 2004. Thus, in one iteration of the Strait and Grine character matrix (hereafter, S\&G-separate) there is a 'pre-2004 A. anamensis' OTU that is composed of specimens that could be scored for dentognathic characters but not for most cranial characters. We added a separate OTU for MRD, which could be scored for cranial characters but not for most dental and mandibular characters. In another iteration (S\&G-combined), the samples are combined into a single 'MRD-A. ana-mensis' OTU. With regard to the few characters that could be scored for both the pre-2004 A. anamensis and MRD samples, the scorings were either consistent between samples or could be accommodated by a pre-existing category of 'variable' (used to represent a polymorphic population). We conducted comparable analyses using the matrix of Kimbel et al. ${ }^{27}$ one iteration treating MRD as its own taxo-nomic unit (K-separate) and the second combining MRD and previously attributed $A$. anamensis specimens (K-combined). Because the previous analysis ${ }^{27}$ did not include A. anamensis, our scorings for A. anamensis in the K-combined matrix are based on first-hand comparison of the original fossils, together with published descriptions and comparative data.

The varied OTU treatment of MRD and other specimens attributed to A. anamensis allows the phylogenetic placement of MRD to be assessed with or without the assumptions implied by our species attribution. Our alpha taxonomic designation is embedded in the K-combined and S\&G-combined analyses-the MRD cranial features are associated with the dentognathic features of the pre-2004 A. anamensis sample and any differences between the samples are taken to represent intraspecific polymorphism. By contrast, these assumptions are avoided in the Kseparate and S\&G-separate analyses, in which MRD can be understood as a separate site sample, a subspecies or as a different species. Assuming that the conspecific designation is correct, the OTU separation further permits us to assess whether different character subsets (for example, cranial versus dentognathic) convey consistent information about the phylogenetic placement of $A$. anamensis. 
a
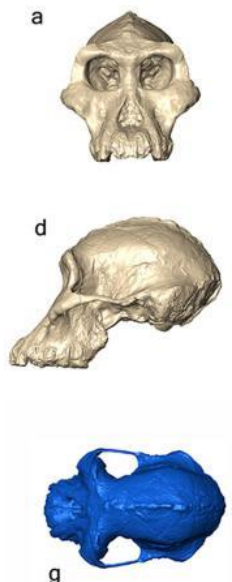

b
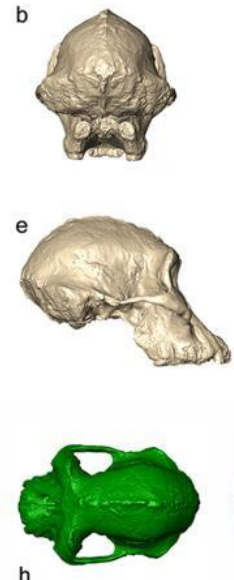

$\mathrm{h}$

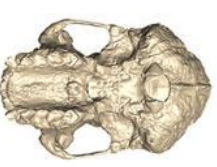

C
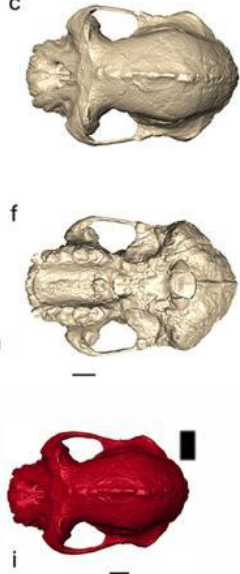

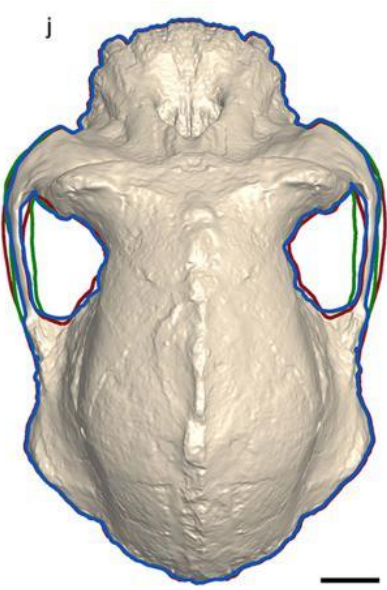

Extended Data Fig. 1 | MRD-VP-1/1 digital reconstructions. Digitally reconstructed cranium and comparison of three alternative reconstructions. a-f, The reconstructed cranium MRD- Sts 5 is shown in anterior view (a), posterior view (b), superior view (c), left (d) and right (e) lateral views and inferior view (f). Additional images illustrate minor

differences in reconstructed zygomatic arches. g, MRD-Sts 5. h, MRD-A.L. 444-2. i, MRD-WT 17000. j, MRD-Sts 5 with edges superimposed to the three different restored versions: MRD- Sts 5 (blue lines); MRD-A.L. 444-2 (green lines) and MRD-WT 17000 (red lines). Scale bars, $2 \mathrm{~cm}$. 

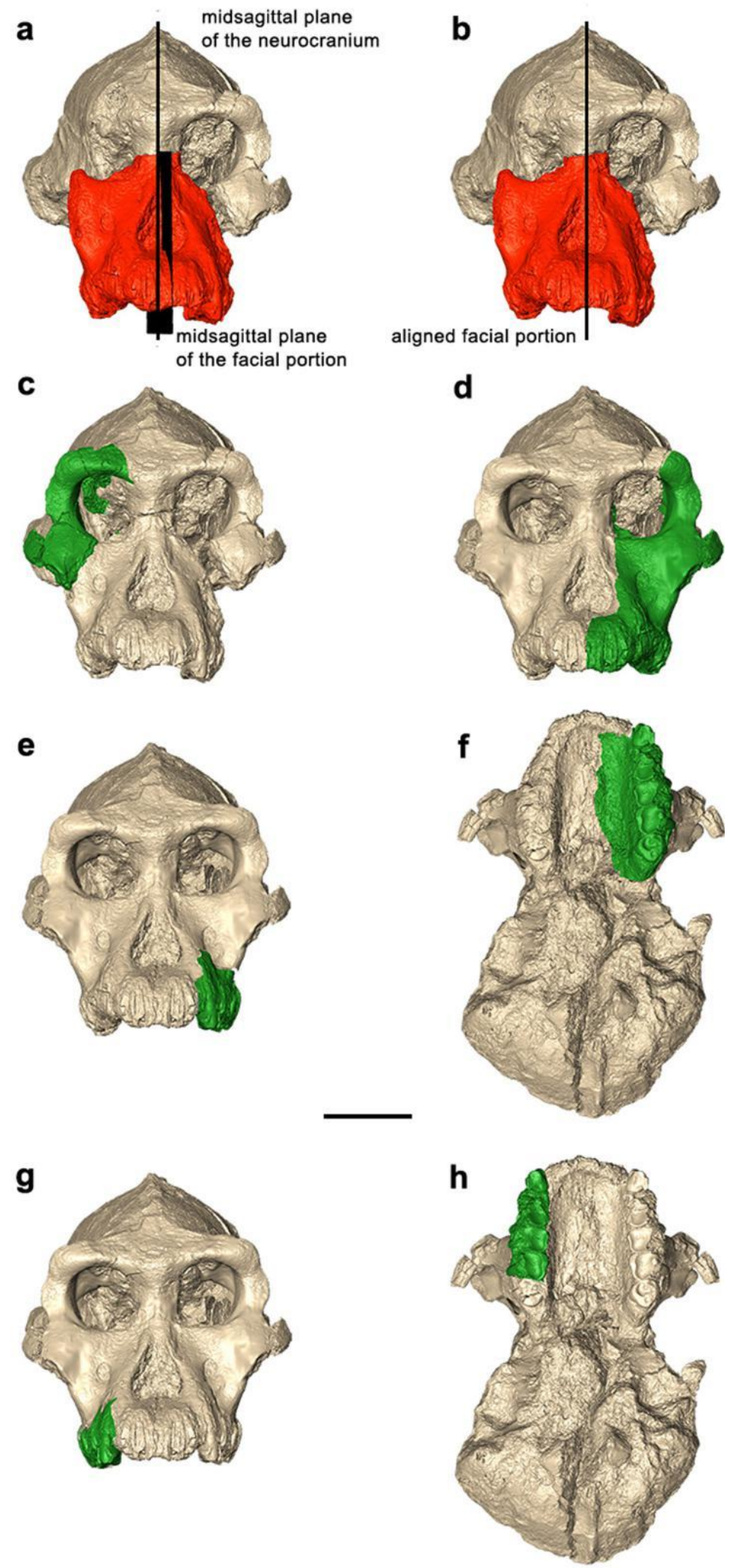

Extended Data Fig. 2 | Basic steps involved in repositioning and reconstructing the MRD face. a, Midsagittal planes computed for the original neurocranium (ivory) and facial portion (red). b. The original facial portion is rotated to align the midsagittal plane of the face with the midsagittal plane of the neurocranium, then the former is moved along the midsagittal plane to establish contact with the latter. c, The left supra-orbital bone was mirrored and aligned to the original MRD

right side. d, The complete right side was mirrored and aligned to the left side. e, f, Anterior (e) and inferior ( $\mathbf{f}$ ) views of the original left maxillary dental arcade and part of the palate superimposed to the mirrored copy of the right hemiface. $\mathbf{g}, \mathbf{h}$, Frontal $(\mathbf{g})$ and basal ( $\mathbf{h})$ view of the right dental arcade reconstructed by mirroring the left dental arcade (except for the right $\mathrm{M}^{3}$ ). Mirrored portions are shown in green. Scale bar, $4 \mathrm{~cm}$. See Methods for details. 

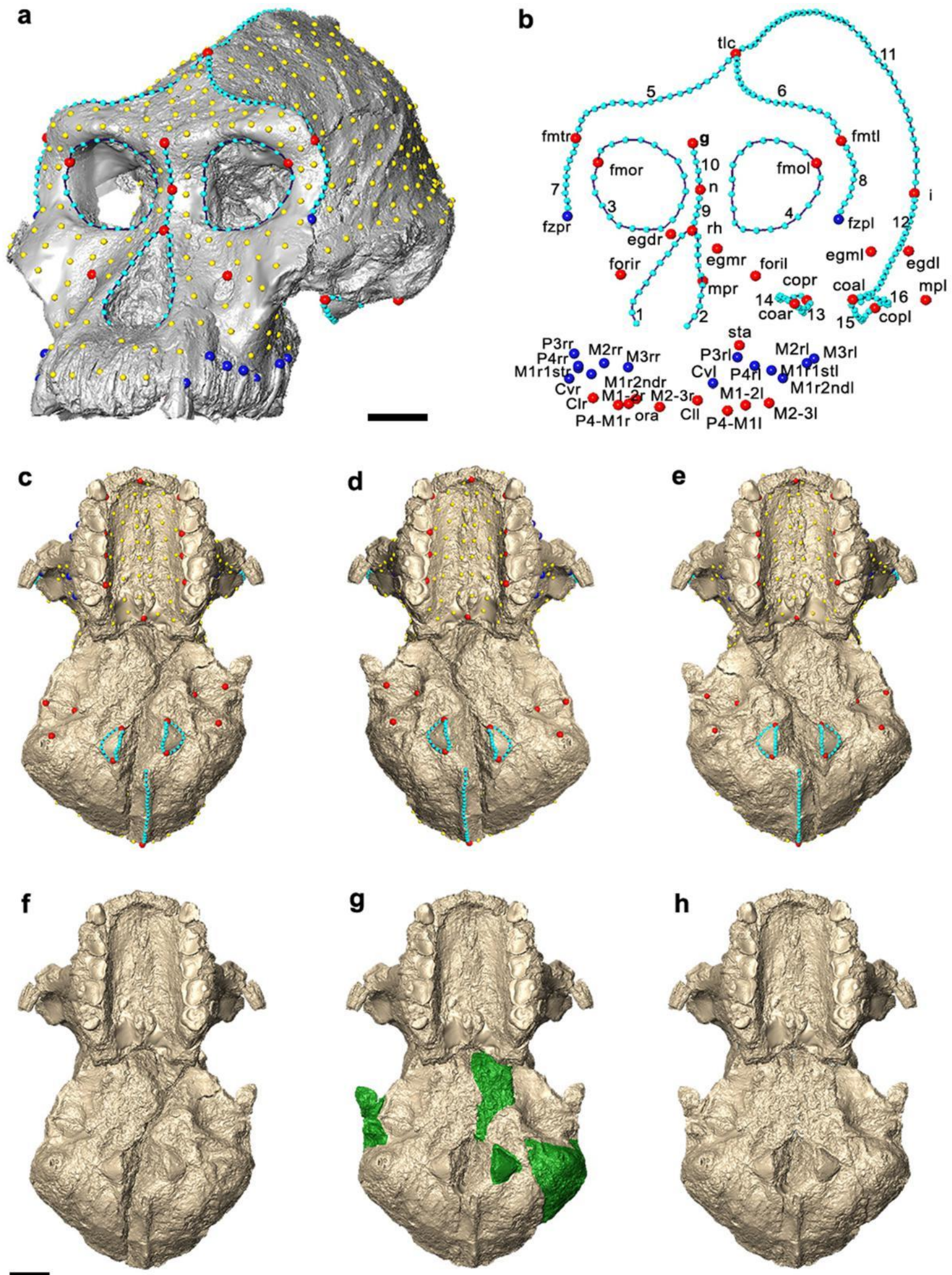

Extended Data Fig. $3 \mid$ MRD symmetrization using a reflected relabelling procedure and neurocranium reconstruction. a, The template with landmarks (red), non-osteometric homologous landmarks (blue), curve semilandmarks (light blue) and surface semilandmarks (yellow) was digitized on the MRD cranium. $\mathbf{b}$, The template configuration with names of landmarks and curves numbers (Supplementary Note 2). c, Basal view of the template digitized on the MRD cranium. $\mathbf{d}$, The template digitized on the mirrored cranium. e, Symmetric configuration of the (semi)landmarks and warped surface. f, Basal view of the final result for MRD- sym. g, Basal view of the left zygomatic process, the right mastoid process and other parts of the basicranium reconstructed by mirroring the original counterparts (integrated parts are shown in green). $\mathbf{h}$, Basal view, final result after integrating the mirrored counterparts. Scale bars, $2 \mathrm{~cm}$. See Methods for details. 

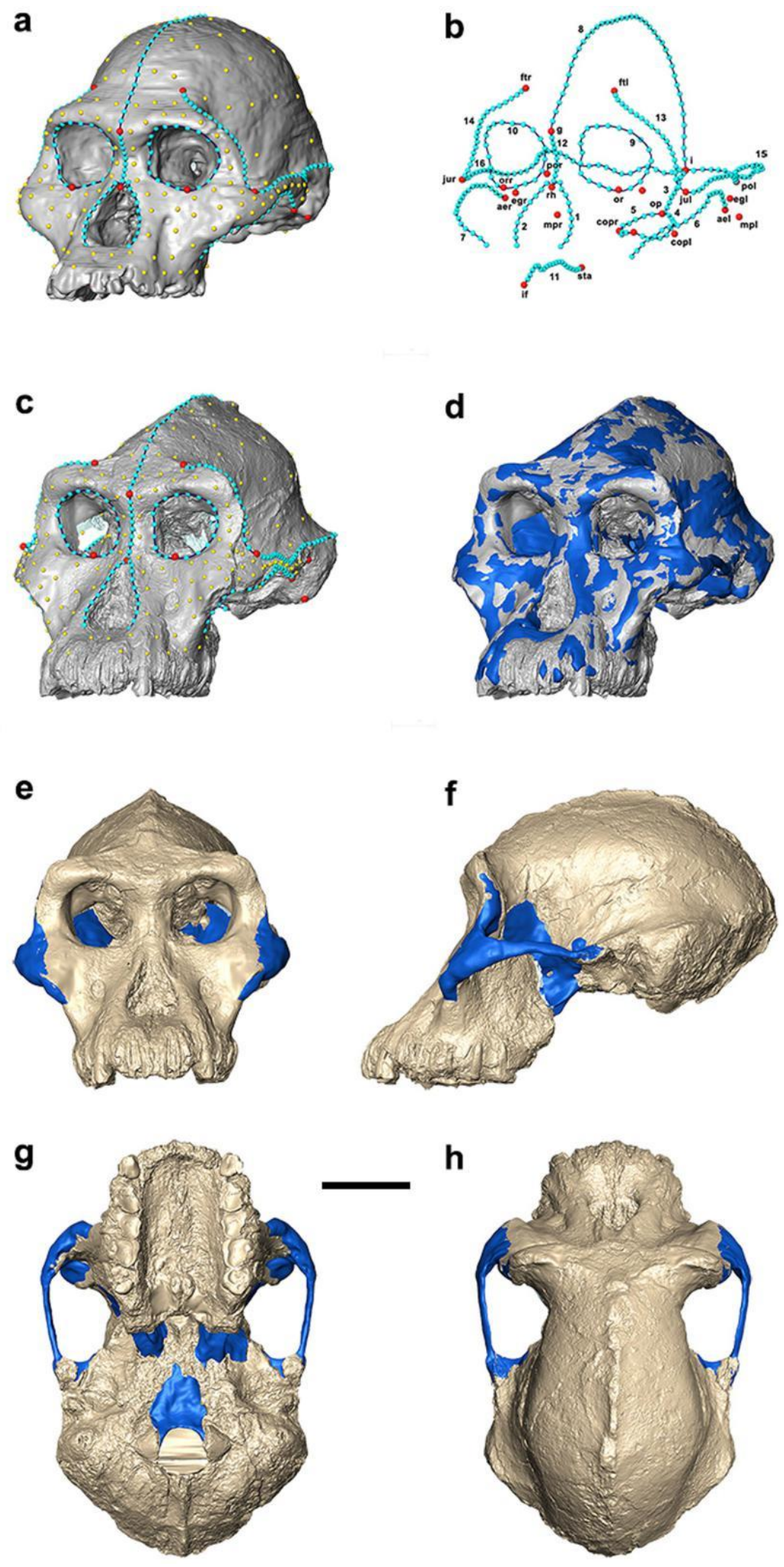

Extended Data Fig. $4 \mid$ Integration of missing parts using Sts 5. a Template built on the cranium of A. africanus (Sts 5). Templates with landmarks (red), curve semilandmarks (light blue) and surface semilandmarks (yellow) were digitized on Sts 5. b. Template configuration with names of landmarks and curves numbers (labels are related to Supplementary Note 3). c, The same set of (semi)landmarks on the MRD-

sym cranium. d, TPS interpolation of the Sts 5 cranium, warped to MRD-sym (blue and grey, respectively). $\mathbf{e}-\mathbf{h}$, MRD-sym with the integrated missing parts (blue) isolated from the resulting warped surfaces obtained by the deformation of Sts 5 (shown here as an example) in anterior (e), left lateral (f), inferior (g) and superior (h) views. Scale bar, $4 \mathrm{~cm}$. 
a
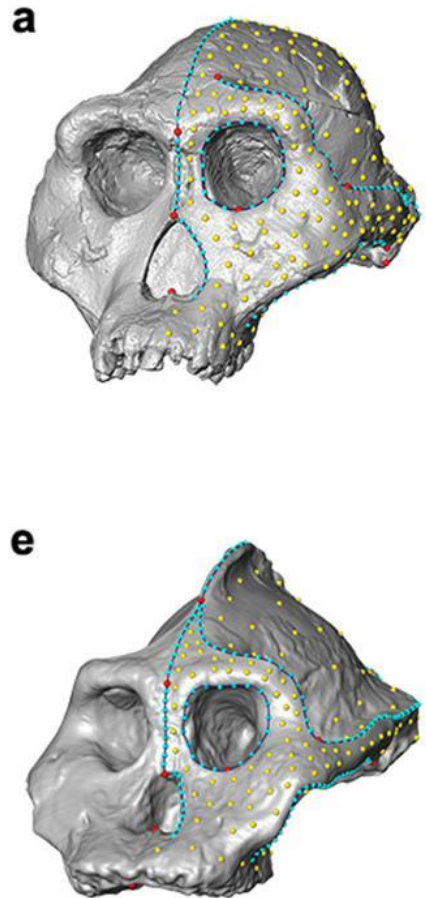

b

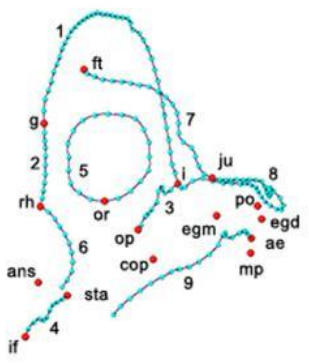

C

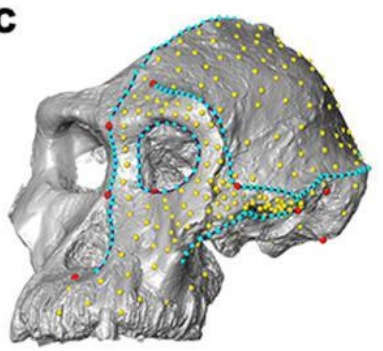

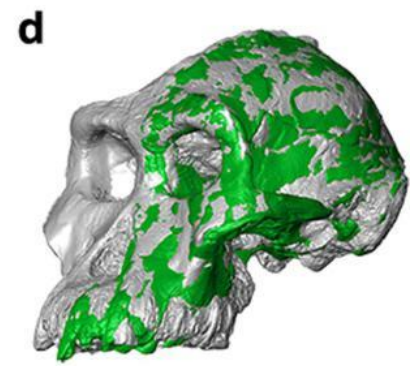

f

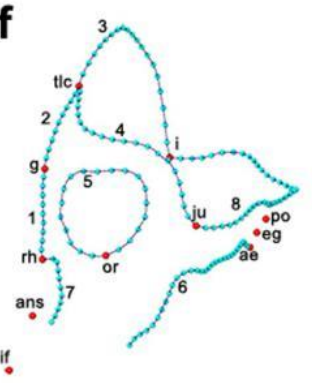

it.

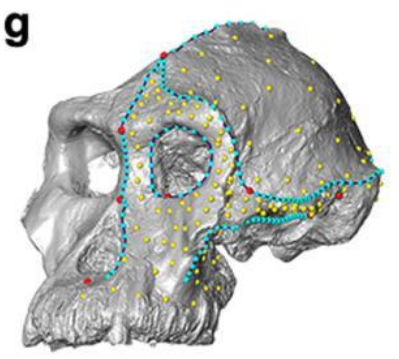

h

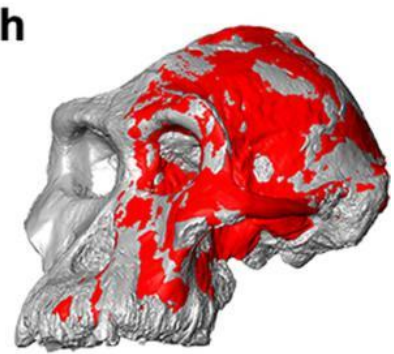

Extended Data Fig. 5 | Integration of missing parts using A.L. 444-2 and KNM-WT 17000. a, e, Templates with landmarks (red), curve

semilandmarks (light blue) and surface semilandmarks (yellow) digitized on A.L. 444-2 (a) and KNM-WT 17000 (e) crania. b, f, The configurations of (semi)landmarks with names of landmarks and curves numbers

digitized on A.L. 444-2 (b ) and KNM -WT 17000 (f) crania (labels are related to Supplementary Notes 4,5 ). $\mathbf{c}, \mathbf{g}$, Sets of (semi)landmarks on the MRD-sym cranium. d, h, TPS interpolation of the A.L. 444 -2 (green) and KMN-WT 17000 (red) crania warped to MRD-sym (grey). Scale bar, $4 \mathrm{~cm}$. 

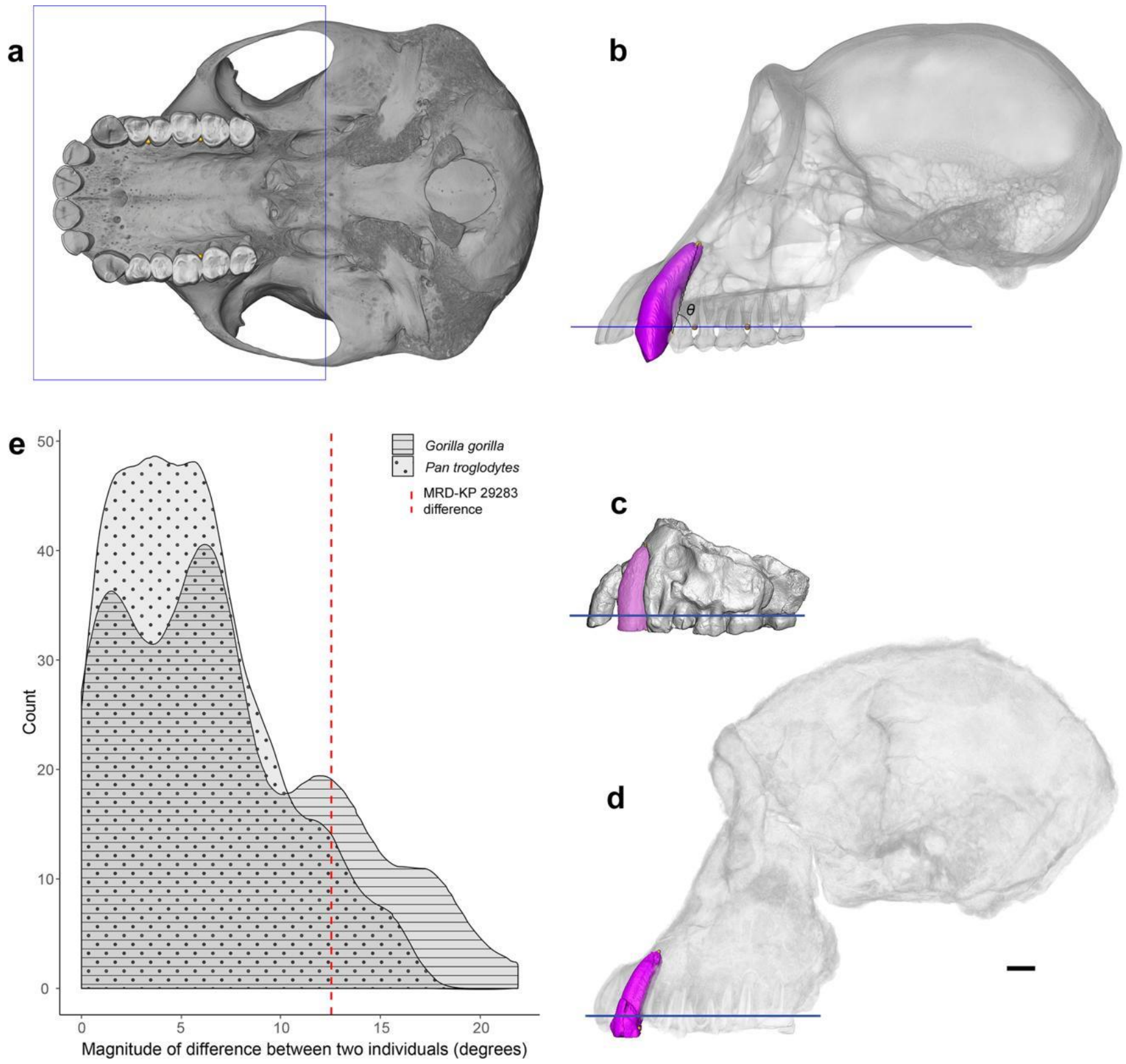

Extended Data Fig. 6 | Canine orientation in MRD and KNM -KP 29283.

The method for measuring the implantation angle of the upper canine is shown in $\mathbf{a}, \mathbf{b}$. a, The internal alveolar plane was established by bestfitting a plane (outlined in blue) to three landmarks between left $\mathrm{P}^{3}$ and $\mathrm{P}^{4}$, between right $\mathrm{P}^{3}$ and $\mathrm{P}^{4}$ and between left $\mathrm{M}^{1}$ and $\mathrm{M}^{2}$. b, Landmarks were placed at the root tip and at the occlusal- most incursion along the distal enamel line. Upper canine implantation was measured in lateral view as the two-dimensional angle $(\theta)$ between a line connecting the canine landmarks and the axis of the alveolar plane. $\mathbf{c}, \mathbf{d}$, Upper canine implantation in the fossil specimens. c, A. anamensis paratype maxilla KNM-KP 29283. d, MRD. Of the other two A. anamensis maxillae

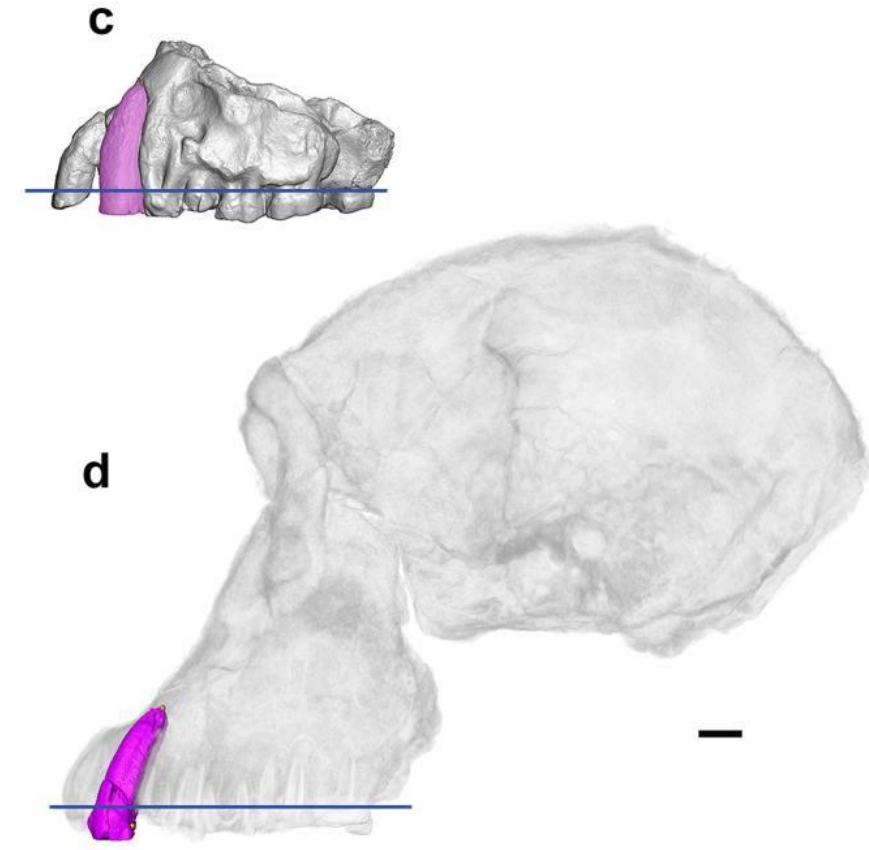

currently known, one (KNM-KP 58579) is qualitatively similar to KNMKP 29283 and the other (ARA-VP- 14/1) is more inclined. Scale bar, $1 \mathrm{~cm}$. The scale bar applies to images in $\mathbf{c}$ and $\mathbf{d}$. e, The magnitude of difference in canine implantation angle between MRD and KNM-KP 29283 (12.5', red dashed line) is shown in the context of expected conspecific differences. The expectation distributions were constructed using a permutation approach, for which the measurements from two individuals were randomly drawn (without replacement) from a comparative sample and the difference in orientation angle was computed. This procedure was repeated 500 times, separately for comparative species $P$. troglodytes and G. gorilla (Supplementary Note 6.2). 

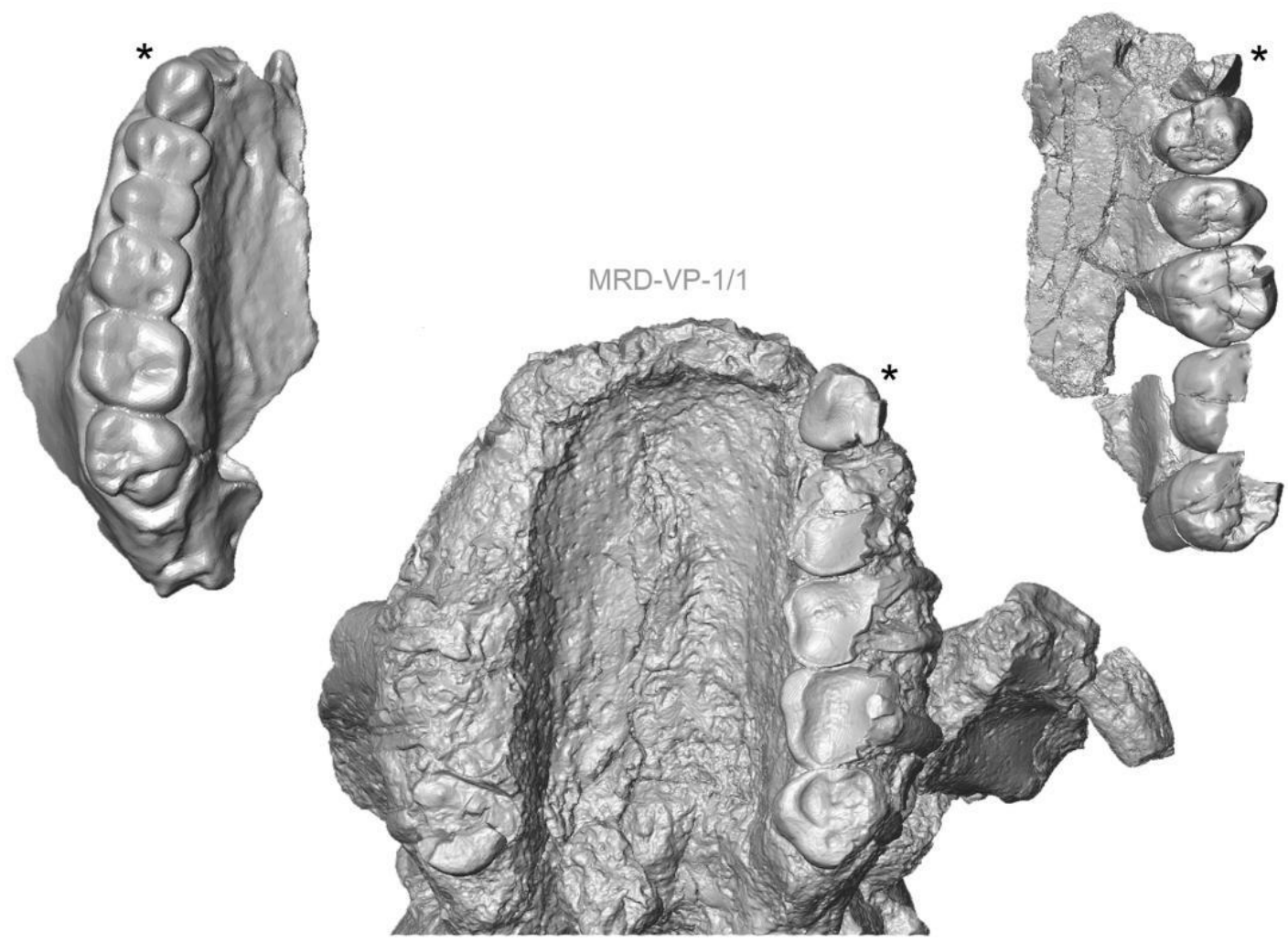

\section{A.L. $444-2$}

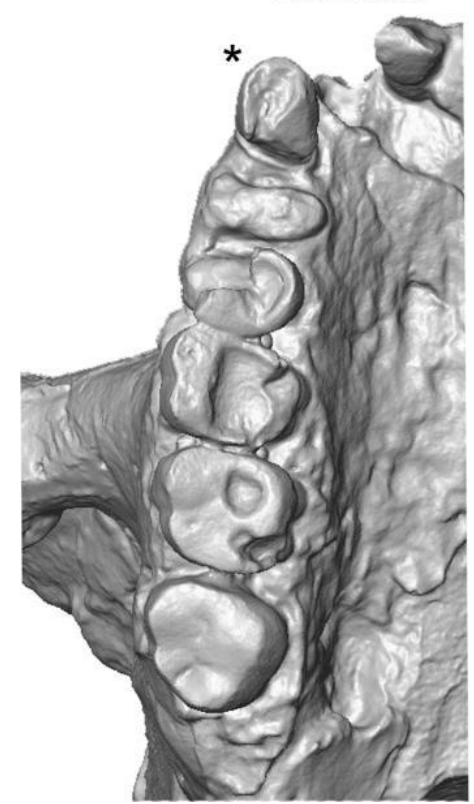

Extended Data Fig. 7 | Maxillary arcade shape. Maxillae in occlusal view of select A. afarensis and A. anamensis specimens and MRD (original, as preserved). The canine and postcanine teeth form a nearly straight line in $A$. anamensis and MRD. By contrast, the canine tends to be slightly
KNM-KP 29283

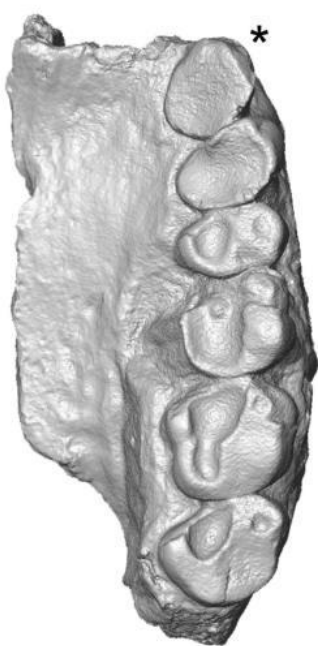

medially offset relative to the postcanine row in many A. afarensis specimens. The position of the canine is indicated by the black asterisk. Scale bar, $1 \mathrm{~cm}$. 
S. tchadensis

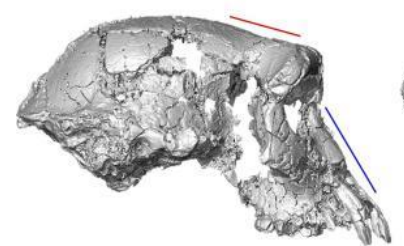

TM 266-01-060-1

recon
A. ramidus

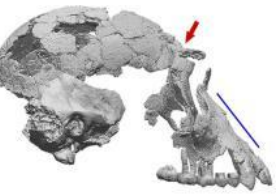

ARA-VP-6/500

composite recon.
MRD-VP-1/1

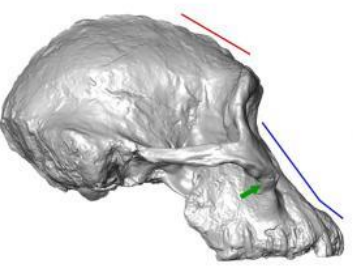

A. afarensis

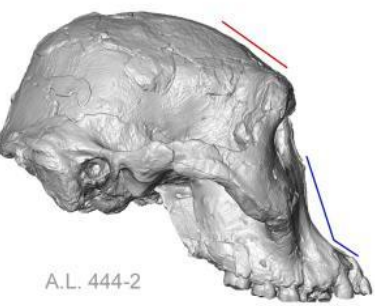

A. africanus

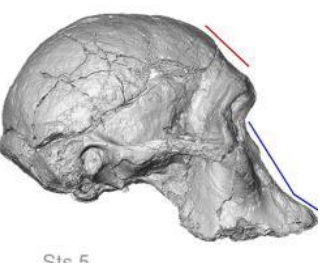

Extended Data Fig. 8 | Comparison of crania in lateral view. Red lines and arrows show the inclination of the frontal and the presence of a post toral sulcus, respectively. Blue lines show the orientation of the mid and lower face, with an broken line indicating a segmented facial profile ${ }^{27}$. The green arrow marks the anterior projection of the zygomatic tubercle (relative to the anterior zygomatic root). Scale bar, $2 \mathrm{~cm}$. 


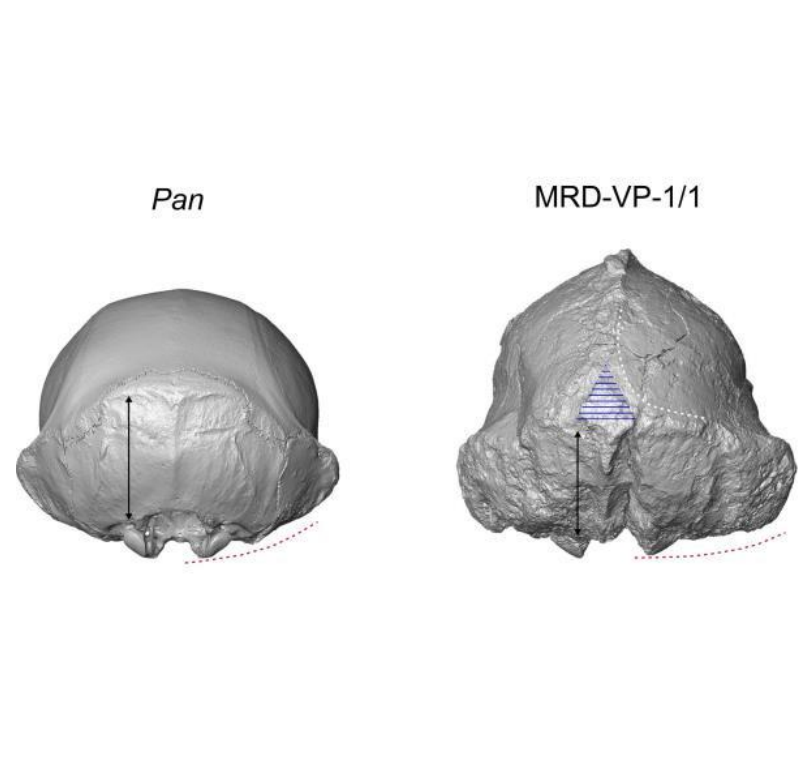

Extended Data Fig. 9 | Comparison of crania in posterior view. The transverse contour of the cranial base is convex in African apes, whereas $A$. afarensis shows an angular transition between the nuchal region and the greatly expanded mastoids (red dashed lines). In this regard, A. afarensis anticipates the morphology of robust australopiths, but $A$. africanus is less derived. MRD shows the primitive convex contour of the base, even though the mastoids are expanded. MRD is also primitive with regard to

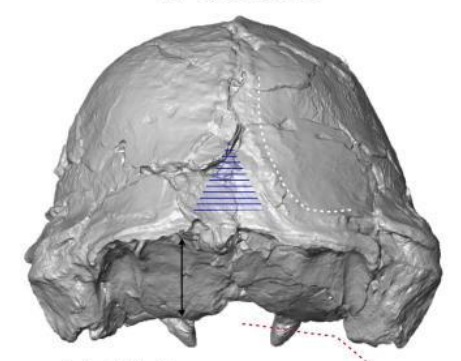

A.L. 444-2

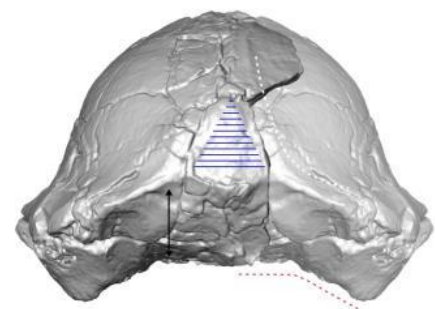

A.L. 333-45 (mirrored left side)

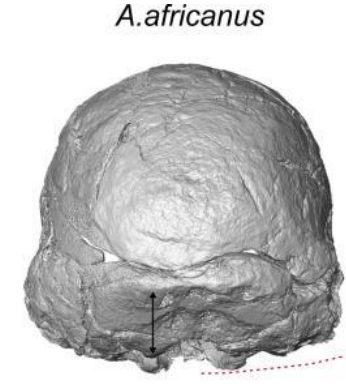

Sts 5

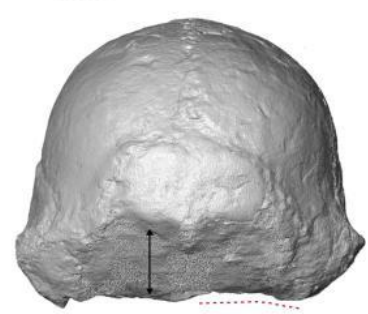

MLD $37 / 38$ the great length of the nuchal plane (black arrows). However, it is similar to A. afarensis in the configuration of the compound temporal-nuchal crest (white dashed lines), the bare area (blue hatched triangle), and the overall 'bell -shaped' posterior outline (that is, the parietal walls are slightly convergent superiorly and the greatest width occurs basally across the enlarged mastoids). 

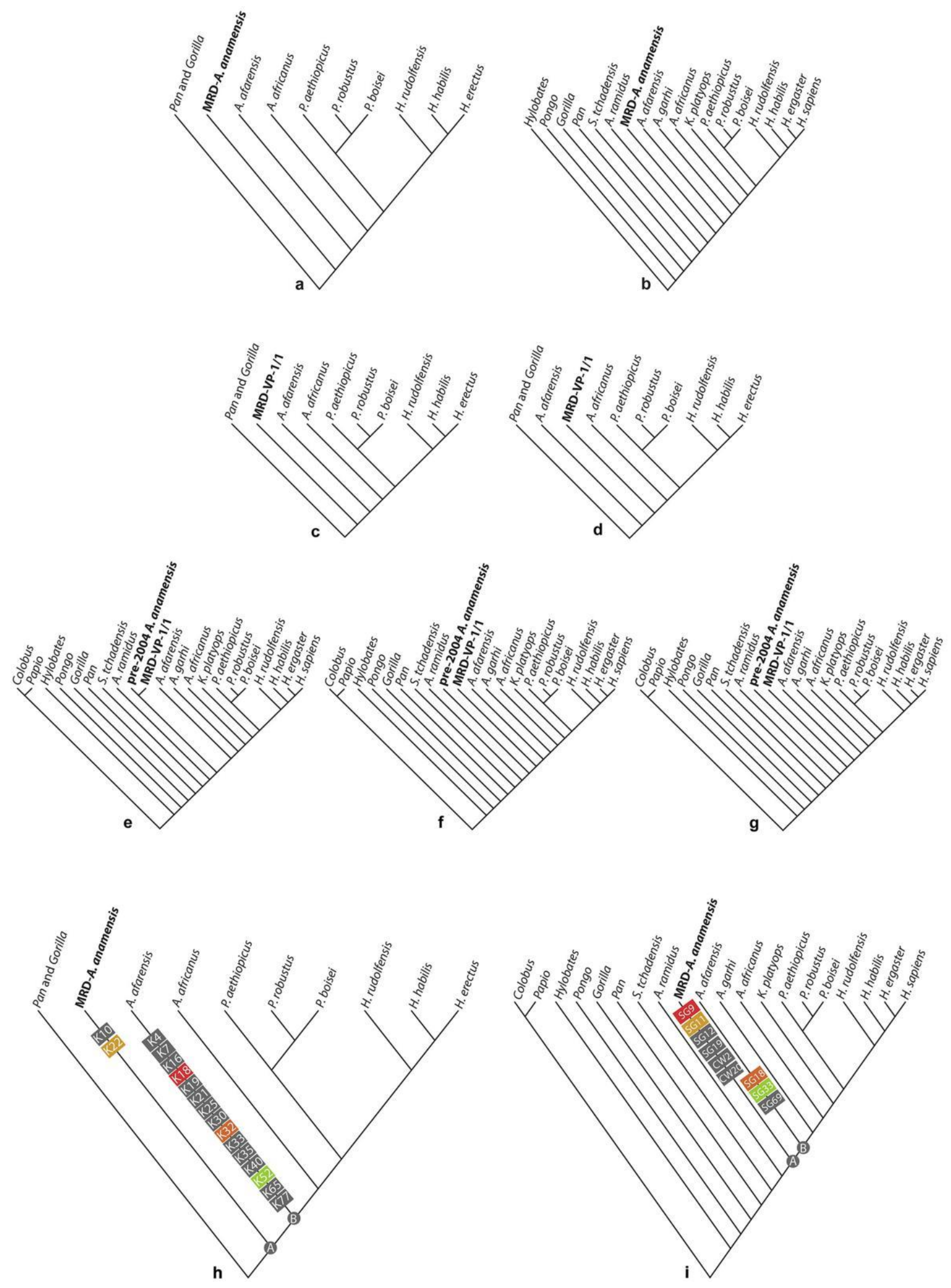

Extended Data Fig. 10 | See next page for caption. 
Extended Data Fig. 10 | Results of phylogenetic analyses. a, Cladogram resulting from the character matrix of ref. ${ }^{27}$, with the addition of MRD and previously described A. anamensis specimens (combined as a single OTU, $\mathrm{K}$ -combined). Parsimony analysis returned a single most-parsimonious tree $(l=$ $196, C=0.71, R=0.70)$. $\mathbf{b}$, Cladogram resulting from the character matrix of ref. ${ }^{33}$ (and references therein) with the addition of the combined MRD- $A$. anamensis OTU (S\&G-combined). This analysis returned a single mostparsimonious tree $(l=429, C=0.47, R=0.66)$ with identical topology. The position of the combined MRD-A. anamensis OTU reinforces accepted relationships and is consistent with geochronology. $\mathbf{c}-\mathbf{g}$, Cladograms resulting from analyses in which MRD is treated as a separate OTU (that is, an OTU bearing observations primarily for cranial characters, but very few dental characters and no mandibular characters.) $\mathbf{c}, \mathbf{d}$, Equally parsimonious cladograms from the K-separate analysis $(l=196, C=0.71, R=0.68)$. e-g, Equally parsimonious cladograms from the $\mathrm{S} \& \mathrm{G}$-separate analysis $(l=430, C=0.47, R=0.66)$. The 'pre-2004 A. anamensis' OTU in e-g bears observations primarily on dentognathic characters. Character scores for MRD are provided in Supplementary Table 1, sheets 1 and 2. Regardless of whether cranial or dentognathic characters are considered, the phylogenetic placement of MRD and the previously known A. anamensis sample remains stable relative to other hominins. $\mathbf{h}, \mathbf{i}$, Cladograms from the K-combined and S\&G-combined analyses (as in $\mathbf{a}$ and $\mathbf{b}$ ), with apomorphies added to the cladograms to illustrate the implied pattern of evolutionary change. The character states reconstructed at nodes A and B provide the reference for identifying $A$. anamensis and A. afarensis apomorphies, which are shown here as rectangles containing their abbreviated character labels. Characters in red, orange, gold and green describe similar morphology and appear in both previously published studies ${ }^{27,33}$. See Supplementary Note 9 and Supplementary Table 1. 